JOURNAL OF THE

AMERICAN MATHEMATICAL SOCIETY

Volume 13, Number 2, Pages 433-466

S 0894-0347(00)00327-1

Article electronically published on January 31, 2000

\title{
COADJOINT ORBITS, MOMENT POLYTOPES, AND THE HILBERT-MUMFORD CRITERION
}

\author{
ARKADY BERENSTEIN AND REYER SJAMAAR
}

\section{Contents}

1. Introduction

2. Subgroups and Weyl chambers 435

3. Main results 445

4. Semistability 452

5. Examples 456

$\begin{array}{lll}\text { Appendix A. Flag varieties } & 462\end{array}$

Appendix B. Notation $\quad 464$

References

\section{INTRODUCTION}

Let $K$ be a compact connected Lie group and let $\tilde{K}$ be a closed connected subgroup. Let $f$ denote the inclusion of $\tilde{K}$ into $K, f_{*}: \tilde{\mathfrak{k}} \rightarrow \mathfrak{k}$ the induced embedding of Lie algebras, and $f^{*}: \mathfrak{k}^{*} \rightarrow \tilde{\mathfrak{k}}^{*}$ the dual projection. In this paper we present a solution to the following problem.

(i) Let $\mathcal{O}$ be a coadjoint orbit of $K$. Its projection $f^{*}(\mathcal{O})$ is a $\tilde{K}$-stable subset of $\tilde{\mathfrak{k}}^{*}$. Which coadjoint orbits of $\tilde{K}$ are contained in $f^{*}(\mathcal{O})$ ?

Select maximal tori $T$ in $K$ and $\tilde{T}$ in $\tilde{K}$, and Weyl chambers $\mathfrak{t}_{+}^{*}$ in $\mathfrak{t}^{*}$ and $\tilde{\mathfrak{t}}_{+}^{*}$ in $\tilde{\mathfrak{t}}^{*}$, where $\mathfrak{t}$ and $\tilde{\mathfrak{t}}$ denote the Lie algebras of $T$, resp. $\tilde{T}$. The set $f^{*}(\mathcal{O})$ is completely determined by the intersection $\Delta(\mathcal{O})=f^{*}(\mathcal{O}) \cap \tilde{\mathfrak{t}}_{+}^{*}$, and according to a result of Heckman [12], $\Delta(\mathcal{O})$ is a convex polytope. It can be described as the support of the asymptotic multiplicity function, for which Heckman found an explicit formula. However, because the formula involves an alternating sum in which many cancellations take place, it is in practice impossible to determine the polytope from it. Our goal is to write down as explicitly as possible the inequalities defining the polytope.

Our results are inspired by a remarkable paper of Klyachko 15], in which he solved the case of the diagonal embedding of $\mathbf{U}(n)$ into $\mathbf{U}(n) \times \mathbf{U}(n)$. See also Fulton's recent survey paper [9. As in [15], the proof of our inequalities relies on

Received by the editors April 30, 1999 and, in revised form, November 21, 1999.

2000 Mathematics Subject Classification. Primary 53D20; Secondary 14L24.

The second author was partially supported by an Alfred P. Sloan Research Fellowship and by NSF Grant DMS-9703947. 
the Hilbert-Mumford criterion for semistability of flags with respect to equivariant ample line bundles. A related result describing the "general" faces of the polytope was arrived at independently by Brion [5].

Recall that the restriction of $f^{*}$ to $\mathcal{O}$ is nothing but the moment map for the action of $\tilde{K}$ on the homogeneous symplectic $K$-manifold $\mathcal{O}$ and that $\Delta(\mathcal{O})$ is its moment polytope. (See Kirwan [14.) Observe also that $\mathcal{O}$ is a symplectic quotient of the cotangent bundle $T^{*} K$. This implies that $\Delta(\mathcal{O})$ is equal to the intersection of $\Delta\left(T^{*} K\right)$ with an affine subspace, where $\Delta\left(T^{*} K\right)$ denotes the moment cone of $T^{*} K$ with respect to the $\tilde{K} \times K$-action. We shall in fact write a complete set of inequalities for the cone $\Delta\left(T^{*} K\right)$.

We do so in terms of the Schubert bases of the cohomology groups $H^{\bullet}(X)$ and $H^{\bullet}(\tilde{X})$, the canonical homomorphism $\phi: H^{\bullet}(X) \rightarrow H^{\bullet}(\tilde{X})$, and the action on $H^{\bullet}(X)$ of a certain subset of the Weyl group of $K$, which we name the relative Weyl set. Here $X$ and $\tilde{X}$ denote the flag varieties of $K$ and $\tilde{K}$, respectively. Determining the relative Weyl set and the matrix of $\phi$ relative to the Schubert bases are interesting combinatorial problems, which we do not know how to solve in general. In many examples, however, they can be solved and we obtain explicit inequalities for the moment cone. We treat a few of these examples in detail.

An intriguing multiplicative version of Klyachko's theorem was obtained by Agnihotri and Woodward [1] and Belkale [2], who considered conjugacy classes in $\mathbf{U}(n)$ and found eigenvalue inequalities for products of unitary matrices in terms of quantum Schubert calculus. An interesting question is to what extent their results can be generalized to the setting of the present paper.

Heckman showed that the geometric problem (ii) is closely related to the following algebraic problem.

(ii) Let $V$ be an irreducible $K$-module. As a $\tilde{K}$-module it breaks up into isotypical components. Which irreducible $\tilde{K}$-modules occur in $V$ ?

Indeed, assume that $\mathcal{O}$ is integral in the sense that it is the orbit through an integral point $\lambda \in \mathfrak{t}_{+}^{*}$ and that $V_{\lambda}$ is the irreducible $K$-module with highest weight $\lambda$. Likewise, let $\tilde{V}_{\tilde{\lambda}}$ be the irreducible $\tilde{K}$-module attached to an integral coadjoint $\tilde{K}$-orbit $\tilde{\mathcal{O}}$. Then if $\tilde{V}_{\tilde{\lambda}}$ occurs in $V_{\lambda}, \tilde{\mathcal{O}}$ must be contained in $f^{*}(\mathcal{O})$. (The converse question - which integral $\tilde{K}$-orbits inside $f^{*}(\mathcal{O})$ correspond to irreducible components of $V_{\lambda}$ ? - is in general much harder, and will be discussed only briefly in this paper.) As was pointed out by Guillemin and Sternberg [10], Heckman's work implies that the following asymptotic version of (iii) is equivalent to (ii).

(ii') For which rational points $(\tilde{\lambda}, \lambda)$ in $\tilde{\mathfrak{t}}_{+}^{*} \times \mathfrak{t}_{+}^{*}$ does there exist a positive integer $n$ such that $(n \tilde{\lambda}, n \lambda)$ is integral and the irreducible module $\tilde{V}_{n \tilde{\lambda}}$ occurs in $V_{n \lambda}$ ?

Let $\mathcal{S}$ denote the set of all pairs of dominant weights $(\tilde{\lambda}, \lambda)$ such that $\tilde{V}_{\tilde{\lambda}}$ occurs in $V_{\lambda}$, and let $\mathcal{S}^{\prime}$ denote the set of all pairs $(\tilde{\lambda}, \lambda)$ in $\tilde{\mathfrak{t}}_{+}^{*} \times \mathfrak{t}_{+}^{*}$ which satisfy (ii'). Krämer proved in [17] that $\mathcal{S}$ is closed under addition. Therefore $\mathcal{S}^{\prime}$ is equal to the convex hull over the rationals of $\mathcal{S}$. The correspondence between problems (ii) and (ii) is then as follows: $\mathcal{S}^{\prime}$ is equal to the set of all rational points inside $\Delta\left(T^{*} K\right)$, and $\Delta\left(T^{*} K\right)$ is equal to the closure of $\mathcal{S}^{\prime}$. (See e.g. [22, Theorem 7.6].) This correspondence plays an important role in the proof of our main result.

In the remainder of this paper we will, for convenience, allow $f$ to be a homomorphism with finite kernel. This does not change the nature of problems (i) and 
(ii') in any way, because the sets $\mathcal{S}$ and $\Delta\left(T^{*} K\right)$ depend only on the Lie algebras of $K$ and $\tilde{K}$.

Section 2 is a collection of prerequisite results on subgroups and Weyl chambers. The main result of this paper, Theorem 3.1.1, is stated in Section 3 and proved in Section 4 Section 5 contains the examples. In Appendix $\mathrm{A}$ we review some material on flag varieties and Appendix $B$ is a compendium of our notational conventions.

We are grateful to Friedrich Knop for a number of helpful comments.

\section{Subgroups and Weyl Chambers}

Throughout this paper $\tilde{K}$ and $K$ denote compact connected Lie groups and $f: \tilde{K} \rightarrow K$ denotes a Lie group homomorphism with finite kernel. We fix once and for all maximal tori $T$ of $K$ and $\tilde{T}$ of $\tilde{K}$ such that $\tilde{T}=T \cap \tilde{K}$. This section covers a number of auxiliary results which are needed in Section 3 to state our main result. Some are elementary, but are included for lack of a reference. In Section 2.1 we show that the Weyl group of $\tilde{K}$ can be viewed as a subgroup of the Weyl group of $K$ in a number of different ways. In Section 2.2 we discuss how the partition of the Cartan subalgebra $\mathfrak{t}=\operatorname{Lie} T$ into Weyl chambers induces a partition of the small Cartan $\tilde{\mathfrak{t}}=$ Lie $\tilde{T}$ which refines its partition into Weyl chambers. In Section 2.3 we work out an example.

For the purpose of understanding Section 3 the most important parts of this section are 2.2.2 2.2.8. On a first reading the reader may want to skim through these and through Example [2.3.2, and then move on to Section 3 .

A comment on our notation: whenever $O$ is any object or structure associated with the group $K$ in a natural way, the corresponding object or structure associated with $\tilde{K}$ is designated by $\tilde{O}$.

2.1. The Weyl groups. The main result of this section is Theorem 2.1.4 which says that there is a canonically defined family of injective homomorphisms from $\widetilde{W}$ into $W$, which is parametrized by a certain subgroup $\bar{W}$ of $W$. Because the homomorphism $f$ has finite kernel, $\tilde{K}$ and $f(\tilde{K})$ have the same Weyl groups. For this reason, let us assume in this section that $f$ is injective and identify $\tilde{K}$ with $f(\tilde{K})$.

If $K$ acts on a set $M$, then the normalizer of a subset $M^{\prime}$ is the subgroup $\mathcal{N}_{K}\left(M^{\prime}\right)=\left\{k \in K \mid k M^{\prime}=M^{\prime}\right\}$. We say that a subset $K^{\prime}$ of $K$ normalizes $M^{\prime}$ if $K^{\prime} \subseteq \mathcal{N}_{K}\left(M^{\prime}\right)$. The centralizer of $M^{\prime}$ is the subgroup $\mathcal{Z}_{K}\left(M^{\prime}\right)=\bigcap_{m \in M^{\prime}} K_{m}$, which is normal in $\mathcal{N}_{K}\left(M^{\prime}\right)$. (Here $K_{m}$ denotes the stabilizer or isotropy subgroup of $m$.) We will call the quotient

$$
W_{K}\left(M^{\prime}\right)=\mathcal{N}_{K}\left(M^{\prime}\right) / \mathcal{Z}_{K}\left(M^{\prime}\right)
$$

the Weyl group of $K$ relative to $M^{\prime}$. Taking $M=K$, acting on itself by conjugation, we have a Weyl group $W_{K}\left(K^{\prime}\right)$ relative to any subset $K^{\prime}$ of $K$. In particular, the Weyl group of $K$ is $W=W_{K}(T)$.

2.1.1. Lemma. (i) Let $E$ and $F$ be closed subgroups of $K$ and let $E$ act on $K$ by conjugation. If $E$ normalizes $F$, then it also normalizes the commutator subgroup $[F, F]$, the centre $C(F)$, the centralizer $\mathcal{Z}_{K}(F)$, and the identity component $F^{0}$. 
(ii) Let $T^{\prime}$ be any closed subgroup of $T$ and let $L=\mathcal{Z}_{K}\left(T^{\prime}\right)$. Then

$$
\begin{gathered}
\mathcal{N}_{K}(L)=L \cdot\left(\mathcal{N}_{K}(L) \cap \mathcal{N}_{K}(T)\right)=L^{0} \cdot\left(\mathcal{N}_{K}(L) \cap \mathcal{N}_{K}(T)\right), \\
\mathcal{N}_{K}\left(T^{\prime}\right)=L \cdot\left(\mathcal{N}_{K}\left(T^{\prime}\right) \cap \mathcal{N}_{K}(T)\right)=L^{0} \cdot\left(\mathcal{N}_{K}\left(T^{\prime}\right) \cap \mathcal{N}_{K}(T)\right),
\end{gathered}
$$

and the inclusion $\mathcal{N}_{K}\left(T^{\prime}\right) \cap \mathcal{N}_{K}(T) \hookrightarrow \mathcal{N}_{K}\left(T^{\prime}\right)$ induces an isomorphism

$$
\mathcal{N}_{W}\left(T^{\prime}\right) / \mathcal{Z}_{W}\left(T^{\prime}\right) \longleftrightarrow W_{K}\left(T^{\prime}\right) .
$$

Hence $W_{K}\left(T^{\prime}\right)$ is finite and the order of $W_{K}\left(T^{\prime}\right)$ divides the order of $W$.

Proof. (ii) is trivial.

It is clear that $\mathcal{N}_{K}(L)$ contains $L \cdot\left(\mathcal{N}_{K}(L) \cap \mathcal{N}_{K}(T)\right)$. Conversely, consider any $g \in \mathcal{N}_{K}(L)$. Then $g T g^{-1} \subseteq L^{0}$ because $L$ contains $T$ and $T$ is connected. But then $g T g^{-1}$ is a maximal torus of $L^{0}$, so there exists $x \in L^{0}$ such that $g T g^{-1}=x T x^{-1}$, or equivalently, $n=x^{-1} g \in \mathcal{N}_{K}(T)$. But then also $n \in L^{0} \cdot \mathcal{N}_{K}(L) \subseteq \mathcal{N}_{K}(L)$. We have shown that $g=x n$ with $x \in L^{0}$ and $n \in \mathcal{N}_{K}(L) \cap \mathcal{N}_{K}(T)$, which proves (2.1). Observe that $\mathcal{N}_{K}\left(T^{\prime}\right) \subseteq \mathcal{N}_{K}(L)$ by (i). Hence, by (2.1), $\mathcal{N}_{K}\left(T^{\prime}\right)$ is contained in the intersection of $L^{0} \cdot\left(\mathcal{N}_{K}(L) \cap \mathcal{N}_{K}(T)\right)$ and $\mathcal{N}_{K}\left(T^{\prime}\right)$, which is equal to $L^{0}$. $\left(\mathcal{N}_{K}\left(T^{\prime}\right) \cap \mathcal{N}_{K}(T)\right)$. The inclusion $L \cdot\left(\mathcal{N}_{K}\left(T^{\prime}\right) \cap \mathcal{N}_{K}(T)\right) \subseteq \mathcal{N}_{K}\left(T^{\prime}\right)$ is obvious. This proves (2.2). The isomorphism (2.3) is Exercice 4 in Bourbaki [4, Ch. 9, §2]. Let us give a hint. It is easy to show that

$$
\begin{gathered}
\mathcal{N}_{W}\left(T^{\prime}\right)=\left(\mathcal{N}_{K}\left(T^{\prime}\right) \cap \mathcal{N}_{K}(T)\right) / T \\
\mathcal{Z}_{W}\left(T^{\prime}\right)=W_{L}(T)=\mathcal{N}_{L}(T) / T=\left(L \cap \mathcal{N}_{K}(T)\right) / T .
\end{gathered}
$$

This implies that $\mathcal{N}_{W}\left(T^{\prime}\right) / \mathcal{Z}_{W}\left(T^{\prime}\right) \cong\left(\mathcal{N}_{K}\left(T^{\prime}\right) \cap \mathcal{N}_{K}(T)\right) /\left(L \cap \mathcal{N}_{K}(T)\right)$. The latter group clearly injects into $W_{K}\left(T^{\prime}\right)$ and it follows from (2.2) that this injection is surjective.

We can apply (2.3) to the maximal torus $\tilde{T}$ of the subgroup $\tilde{K}$. Since $\widetilde{W}$ is a subgroup of $W_{K}(\tilde{T})$ in a natural way, the upshot is that $\tilde{W}$ is a subquotient of $W$. But more is true. Let us denote the subgroup $\mathcal{Z}_{K}(\tilde{T})$ by $\bar{K}$. This is a connected subgroup that contains the maximal torus $T$. Its root system is equal to the subsystem $\bar{R}$ of all roots $\alpha \in R$ which vanish on $\tilde{\mathfrak{t}}$. Alternatively, $\bar{K}$ can be described as the centralizer of $\overline{\mathfrak{s}}: \bar{K}=\mathcal{Z}_{K}(\overline{\mathfrak{s}})=K_{\overline{\mathfrak{s}}}$, where $\overline{\mathfrak{s}}$ is the smallest (closed) face of the Weyl chamber which contains $\tilde{\mathfrak{t}} \cap \mathfrak{t}_{+}$. In other words, $\overline{\mathfrak{s}}$ is the face of $\mathfrak{t}_{+}$ which is perpendicular to $\bar{R}$,

$$
\overline{\mathfrak{s}}=\bigcap_{\alpha \in \bar{R}}\left\{\xi \in \mathfrak{t}_{+} \mid \alpha(\xi)=0\right\} .
$$

Let $C=C(K), \tilde{C}=C(\tilde{K})$ and $\bar{C}=C(\bar{K})$ be the centres of $K, \tilde{K}$ and $\bar{K}$, respectively. Observe that $C \subseteq \bar{C}$ and $\tilde{C} \subseteq \bar{C}^{0}$. The identity component of $\bar{C}$ is the torus whose Lie algebra $\overline{\mathfrak{c}}$ is equal to the linear span of $\overline{\mathfrak{s}}$. For the normalizer and centralizer of $\bar{C}$ we have the following result.

\subsubsection{Lemma.}

$$
\begin{gathered}
\mathcal{Z}_{K}(\bar{C})=\mathcal{Z}_{K}\left(\bar{C}^{0}\right)=\bar{K}, \\
\mathcal{N}_{K}(\bar{C})=\mathcal{N}_{K}\left(\bar{C}^{0}\right)=\mathcal{N}_{K}(\bar{K}), \\
\mathcal{N}_{K}(\tilde{T}) \subseteq \mathcal{N}_{K}(\bar{C}), \\
\mathcal{N}_{\tilde{K}}(\bar{C})=\mathcal{N}_{\tilde{K}}\left(\bar{C}^{0}\right)=\mathcal{N}_{\tilde{K}}(\tilde{T}) .
\end{gathered}
$$


Proof. We have

$$
\mathcal{Z}_{K}(\bar{C}) \subseteq \mathcal{Z}_{K}\left(\bar{C}^{0}\right)=\mathcal{Z}_{K}(\overline{\mathfrak{c}}) \subseteq \mathcal{Z}_{K}(\tilde{\mathfrak{t}})=\mathcal{Z}_{K}(\tilde{T})=\bar{K}
$$

On the other hand, $\bar{C}$ is the centre of $\bar{K}$, so $\bar{K} \subseteq \mathcal{Z}_{K}(\bar{C})$ and therefore all the inclusions in (2.9) are equalities. This proves (2.5). By Lemma 2.1.1(ii) we have $\mathcal{N}_{K}(\bar{K}) \subseteq \mathcal{N}_{K}(\bar{C})$, because $\bar{C}$ is the centre of $\bar{K}$. Likewise, $\mathcal{N}_{K}\left(\bar{C}^{0}\right) \subseteq \mathcal{N}_{K}(\bar{K})$, because $\overline{\bar{K}}$ is the centralizer of $\bar{C}^{0}$, and also $\mathcal{N}_{K}(\bar{C}) \subseteq \mathcal{N}_{K}\left(\bar{C}^{0}\right)$. In sum, we have shown

$$
\mathcal{N}_{K}(\bar{C}) \subseteq \mathcal{N}_{K}\left(\bar{C}^{0}\right) \subseteq \mathcal{N}_{K}(\bar{K}) \subseteq \mathcal{N}_{K}(\bar{C}),
$$

which proves (2.6). The inclusion (2.7) follows from (2.6) plus the fact that $\mathcal{N}_{K}(\tilde{T})$ is contained in $\mathcal{N}_{K}\left(\mathcal{Z}_{K}(\tilde{T})\right)=\mathcal{N}_{K}(\bar{K})$. The inclusion (2.8) follows from (2.6) plus the fact that $\mathcal{N}_{K}(\bar{K}) \cap \tilde{K}=\mathcal{N}_{\tilde{K}}(\tilde{T})$.

This implies the following relationships among the various Weyl groups. Here $\bar{W}=W_{\bar{K}}(T)$ denotes the Weyl group of $\bar{K}$.

2.1.3. Lemma. (i) The inclusions $\mathcal{N}_{\tilde{K}}(\tilde{T}) \subseteq \mathcal{N}_{K}(\tilde{T})$ and (2.7) induce injective homomorphisms

$$
W \hookrightarrow W_{K}(\tilde{T}) \hookrightarrow W_{K}\left(\bar{C}^{0}\right)=W_{K}(\bar{C}) .
$$

(ii) Let $\bar{S}$ be a base (set of simple roots) of the root system $\bar{R}$. Then

$$
\mathcal{N}_{W}(\bar{R}) \cong \mathcal{N}_{W}(\bar{S}) \ltimes \bar{W},
$$

and the projection $\mathcal{N}_{W}(\bar{R}) \rightarrow \mathcal{N}_{W}(\bar{S})$ induces an isomorphism

$$
j_{\bar{S}}: W_{K}(\bar{C}) \longleftrightarrow \mathcal{N}_{W}(\bar{S}) .
$$

Proof. The kernel of the $\operatorname{map} \mathcal{N}_{\tilde{K}}(\tilde{T}) \rightarrow W_{K}(\tilde{T})$ is $\mathcal{Z}_{K}(\tilde{T}) \cap \tilde{K}=\mathcal{Z}_{\tilde{K}}(\tilde{T})=\tilde{T}$, so the induced homomorphism $W \rightarrow W_{K}(\tilde{T})$ is injective. By (2.5), the kernel of the map $\mathcal{N}_{K}(\tilde{T}) \rightarrow W_{K}(\bar{C})$ is $\mathcal{Z}_{K}(\tilde{T})$, so the induced homomorphism $W_{K}(\tilde{T}) \rightarrow W_{K}(\bar{C})$ is injective. This proves (ii).

Observe that $\bar{W}$ is normal in $\mathcal{N}_{W}(\bar{R})$ and that $\mathcal{N}_{W}(\bar{S}) \cap \bar{W}=\{1\}$. If $w \in \mathcal{N}_{W}(\bar{R})$, then $w \bar{S}$ is a base of $\bar{R}$, so $\bar{w} w \bar{S}=\bar{S}$ for some $\bar{w} \in \bar{W}$, which implies $w \in \mathcal{N}_{W}(\bar{S}) \cdot \bar{W}$. This proves (2.11). Inverting the isomorphism (2.3) (with $T^{\prime}=\bar{C}$ ) and using (2.4) yields an isomorphism

$$
W_{K}(\bar{C}) \longleftrightarrow \mathcal{N}_{W}(\bar{C}) / \mathcal{Z}_{W}(\bar{C})=\mathcal{N}_{W}(\bar{C}) / \bar{W} .
$$

We assert that $\mathcal{N}_{W}(\bar{C})=\mathcal{N}_{W}(\bar{R})$. This follows from the observation that $\mathcal{N}_{W}(\bar{C})=$ $\mathcal{N}_{W}\left(\bar{C}^{0}\right)$ is the set of Weyl group elements which preserve $\overline{\mathfrak{c}}$, the intersection of the root hyperplanes defined by $\bar{R}$. Hence we obtain from (2.13) an isomorphism $W_{K}(\bar{C}) \cong \mathcal{N}_{W}(\bar{R}) / \bar{W}$. By 2.11) there is a canonical projection $\mathcal{N}_{W}(\bar{R}) \rightarrow \mathcal{N}_{W}(\bar{S})$, which induces an isomorphism $\mathcal{N}_{W}(\bar{R}) / \bar{W} \cong \mathcal{N}_{W}(\bar{S})$. This proves (2.12).

The base $\bar{S}$ is unique up to an element of $\bar{W}$, and a different choice of base conjugates the isomorphism (2.12): $j_{\bar{w} \bar{S}}(w)=\bar{w} j_{\bar{S}}(w) \bar{w}^{-1}$ for $\bar{w} \in \bar{W}$ and $w \in$ $W_{K}(\bar{C})$. We can summarize these results as follows.

2.1.4. Theorem. A choice of a base $\bar{S}$ of the root subsystem $\bar{R}$ gives rise to a diagram of canonically defined homomorphisms

$$
\widetilde{W} \hookrightarrow W_{K}(\tilde{T}) \longleftrightarrow W_{K}(\bar{C}) \stackrel{j_{\bar{S}}}{\longleftrightarrow} \mathcal{N}_{W}(\bar{S}) \hookrightarrow \mathcal{N}_{W}(\bar{C}) \longleftrightarrow W
$$


Hence the action of $\tilde{W}$ on $\tilde{T}$ extends to an action on the torus $T$ which preserves the intermediate subgroup $\bar{C}$ and the base $\bar{S}$. A different choice of base changes the lifting homomorphism $j_{\bar{S}}$ by conjugation with an element of $\bar{W}=\mathcal{Z}_{W}(\bar{C})$. Therefore the extension of the $\widetilde{W}$-action from $\tilde{T}$ to $\bar{C}$ is unique; the extension from $\bar{C}$ to $T$ is unique up to an element of $\bar{W}$.

Via the isomorphism $j_{\bar{S}}$ we can regard $\widetilde{W}$ as a subgroup of $W$. The image $j_{\bar{S}}(\widetilde{W})$ depends on $\bar{S}$, but the subgroup $j_{\bar{S}}(\widetilde{W}) \bar{W}=\bar{W} j_{\bar{S}}(\widetilde{W})$ does not. We usually abbreviate $j_{\bar{S}}$ to $j$ when the base $\bar{S}$ is understood.

2.1.5. Corollary. The inclusions $\tilde{\mathfrak{t}} \rightarrow \overline{\mathfrak{c}} \rightarrow \mathfrak{t}$ and the dual projections $\mathfrak{t}^{*} \rightarrow \overline{\mathfrak{c}}^{*} \rightarrow \tilde{\mathfrak{t}}^{*}$ are $\widetilde{W}$-equivariant (with respect to any choice of $\bar{S}$ ) and $\bar{W}$-invariant. The set of projected roots $f^{*}(R) \subseteq \tilde{\mathfrak{t}}^{*}$ is $\widetilde{W}$-stable. The embedding $\tilde{K} / \tilde{T} \rightarrow K / T$ induced by $f$ is $\widetilde{W}$-equivariant.

2.1.6. Example. Observe that $\bar{W}=\{1\}$ if and only if $\tilde{K}$ contains a regular element of $K$. This is for instance the case if $\tilde{K}$ is the unit component of the fixed-point group of an automorphism of $K$. (See Bourbaki [4, Ch. 9, §5.3].) If $\bar{W}=\{1\}$, then $\overline{\mathfrak{s}}=\mathfrak{t}_{+}, \bar{K}=T$, and $\bar{C}=\bar{C}^{0}=T$, so (2.14) amounts to

$$
\widetilde{W} \subseteq W_{K}(\tilde{T}) \subseteq W_{K}(T)=\mathcal{N}_{W}(\emptyset)=W,
$$

where the inclusions are canonical.

2.2. The relative Weyl set. The Weyl chambers of the Cartan subalgebra $\mathfrak{t}$ form a simplicial subdivision in the sense that they cover the whole of $\mathfrak{t}$, every chamber is a simplicial cone, and the intersection of any two chambers is a face of each. In this section we study how the subdivision of $\mathfrak{t}$ into chambers induces a subdivision of $\tilde{\mathfrak{t}}$ which is $\widetilde{W}$-invariant and refines the chamber subdivision of $\tilde{\mathfrak{t}}$.

By a face of $\mathfrak{t}$ we mean a closed face of any Weyl chamber in $\mathfrak{t}$. If $\mathfrak{s}$ is a face, let $R_{\mathfrak{s}}$ be the set of all $\alpha \in R$ such that $\alpha \geq 0$ on $\mathfrak{s}$. Let $\mathfrak{g}$ and $\mathfrak{h}$ be the complexifications of $\mathfrak{k}$ and $\mathfrak{t}$, respectively. Then the subalgebra $\mathfrak{p}_{\mathfrak{s}}=\mathfrak{h} \oplus \bigoplus_{\alpha \in R_{\mathfrak{s}}} \mathfrak{g}_{\alpha}$ is parabolic, and the correspondence $\mathfrak{s} \mapsto \mathfrak{p}_{\mathfrak{s}}$ is a bijection between faces of $\mathfrak{t}$ and parabolic subalgebras of $\mathfrak{g}$ containing $\mathfrak{h}$. We denote the relative interior of a polyhedral subset $Q$ of a real vector space by $Q^{\circ}$.

2.2.1. Lemma. (i) $\tilde{R} \subseteq f^{*}(R)$.

Let $\mathfrak{s}$ and $\tilde{\mathfrak{s}}$ be faces of $\mathfrak{t}$ and $\tilde{\mathfrak{t}}$, respectively.

(ii) $\mathfrak{s} \cap \tilde{\mathfrak{t}} \subseteq \tilde{\mathfrak{s}}$ if and only if $(\mathfrak{s} \cap \tilde{\mathfrak{t}})^{\circ} \cap \tilde{\mathfrak{s}}$ is nonempty.

(iii) If $\mathfrak{s} \cap \tilde{\mathfrak{t}} \subseteq \tilde{\mathfrak{s}}$, then $w \mathfrak{s} \cap \tilde{\mathfrak{t}}=\mathfrak{s} \cap \tilde{\mathfrak{t}}$ for all $w \in j\left(\widetilde{W}_{\tilde{\mathfrak{s}}}\right) \bar{W}$, where $\bar{W}$ is the Weyl group of $\bar{K}=\mathcal{Z}_{K}(f(\tilde{T}))$, and $j$ is any lifting homomorphism as in Theorem 2.1.4.

Proof. (ii) follows immediately from the fact that the root-space decomposition $\mathfrak{g}=\mathfrak{h} \oplus \bigoplus_{\alpha \in R} \mathfrak{g}_{\alpha}$ is a refinement of the weight-space decomposition of $\mathfrak{g}$ relative to the subtorus $\tilde{T}$ :

$$
\mathfrak{g}=\overline{\mathfrak{g}} \oplus \bigoplus_{\tilde{\lambda} \in \tilde{\Lambda}^{*} \backslash\{0\}} \mathfrak{g}_{\tilde{\lambda}}
$$

where $\tilde{\Lambda}^{*}$ denotes the weight lattice in $\tilde{\mathfrak{t}}^{*}$ and $\mathfrak{g}_{\tilde{\lambda}}=\bigoplus_{f^{*}(\alpha)=\tilde{\lambda}} \mathfrak{g}_{\alpha}$. 
Let $\mathfrak{s}$ be any face of $\mathfrak{t}$. The subset $\{\alpha \mid \alpha \geq 0$ on $\mathfrak{s} \cap \tilde{\mathfrak{t}}\}$ of $R$ contains $R_{\mathfrak{s}}$ and is therefore of the form $R_{\mathfrak{r}}$, where $\mathfrak{r}$ is a face of $\mathfrak{s}$. It is clear that $\mathfrak{r}$ is the smallest face of $\mathfrak{t}$ which contains $\mathfrak{s} \cap \tilde{\mathfrak{t}}$, and therefore $(\mathfrak{s} \cap \tilde{\mathfrak{t}})^{\circ} \subseteq \mathfrak{r}^{\circ}$.

Now assume that $(\mathfrak{s} \cap \tilde{\mathfrak{t}})^{\circ} \cap \tilde{\mathfrak{s}}$ is nonempty. Pick $\tilde{\lambda} \in(\mathfrak{s} \cap \tilde{\mathfrak{t}})^{\circ} \cap \tilde{\mathfrak{s}}$. Then $\tilde{\lambda} \in \mathfrak{r}^{\circ}$, so a root $\alpha$ is in $R_{\mathfrak{r}}$ if and only if $\alpha(\tilde{\lambda}) \geq 0$. Furthermore $\tilde{\lambda} \in \tilde{\mathfrak{s}}$, so if $\tilde{\alpha} \in \tilde{R}_{\tilde{\mathfrak{s}}}$, then $\tilde{\alpha}(\tilde{\lambda}) \geq 0$. According to (ii) we can write $\tilde{\alpha}=f^{*}(\alpha)$ with $\alpha \in R$. Hence $\alpha(\tilde{\lambda})=\tilde{\alpha}(\tilde{\lambda}) \geq 0$, which implies $\alpha \in R_{\mathfrak{r}}$. Therefore, if $\tilde{\mu}$ is an arbitrary element of $\mathfrak{s} \cap \tilde{\mathfrak{t}}$, then $\tilde{\alpha}(\tilde{\mu})=\alpha(\tilde{\mu}) \geq 0$. This shows that $\mathfrak{s} \cap \tilde{\mathfrak{t}}$ is contained in $\tilde{\mathfrak{s}}$. Conversely, if $\mathfrak{s} \cap \tilde{\mathfrak{t}} \subseteq \tilde{\mathfrak{s}}$, then it is obvious that $(\mathfrak{s} \cap \tilde{\mathfrak{t}})^{\circ} \cap \tilde{\mathfrak{s}}$ is nonempty. This proves (iii).

(iii) follows from the fact that $\bar{W}$ acts trivially on $\tilde{\mathfrak{t}}$ and that $f_{*}$ is $\widetilde{W}$-equivariant.

Select a positive Weyl chamber $\tilde{\mathfrak{t}}_{+}$in $\tilde{\mathfrak{t}}$. Among all chambers of $\mathfrak{t}$, those which intersect $\tilde{\mathfrak{t}}_{+}$in a cone of maximal dimension are of special importance.

2.2.2. Definition. A Weyl chamber $\mathfrak{t}_{+}$in $\mathfrak{t}$ is compatible with $\tilde{\mathfrak{t}}_{+}$if $\operatorname{dim} \tilde{\mathfrak{t}}_{+} \cap \mathfrak{t}_{+}=$ $\operatorname{dim} \tilde{\mathfrak{t}}_{+}$.

Equivalently, $\mathfrak{t}_{+}$is compatible with $\tilde{\mathfrak{t}}_{+}$if there exists a $\tilde{\xi}_{0}$ in the interior of $\tilde{\mathfrak{t}}_{+}$ such that $\alpha(\tilde{\xi}) \geq 0$ for all $\alpha \in S$ and for all $\tilde{\xi} \in \tilde{\mathfrak{t}}$ sufficiently close to $\tilde{\xi}_{0}$. It is obvious that compatible chambers exist: pick an arbitrary set of positive roots $\bar{R}_{+}$ in $\bar{R}$, pick $\tilde{\xi}_{0} \in \tilde{\mathfrak{t}}_{+}^{\circ}$, and let $R_{+}^{\prime}$ be the set of $\alpha \in R$ such that $\alpha\left(\tilde{\xi}_{0}\right)>0$. Then $R_{+}=\bar{R}_{+} \cup R_{+}^{\prime}$ is a set of positive roots, and the chamber in $\mathfrak{t}$ which is positive with respect to $R_{+}$is a compatible chamber. Moreover, every compatible chamber arises in this way.

Henceforth we shall fix chambers $\tilde{\mathfrak{t}}_{+}$and $\mathfrak{t}_{+}$such that $\mathfrak{t}_{+}$is compatible with $\tilde{\mathfrak{t}}_{+}$. We denote the corresponding positive Borel subgroups of $\tilde{G}=\tilde{K}^{\mathbb{C}}$ and $G=K^{\mathbb{C}}$ by $\tilde{B}$ and $B$, respectively.

2.2.3. Remark. This choice entails a choice of bases of the root systems $\tilde{R}$ and $R$, and hence also of a base of $\bar{R}$ and a lifting homomorphism $j$ as in Theorem 2.1.4.

For arbitrary $w \in W$ we define $\tilde{\mathfrak{t}}_{w}$ to be the cone $w \mathfrak{t}_{+} \cap \tilde{\mathfrak{t}}$. The following result gives a set of spanning vectors for the cones dual to $\tilde{\mathfrak{t}}_{w}$ (and hence inequalities for the $\tilde{\mathfrak{t}}_{w}$ ). Let $\mathcal{C}$ denote the cone spanned by the positive roots $R_{+}$. (See Appendix B for our conventions regarding roots and weights.)

2.2.4. Lemma. For all $w \in W$ the cone $\tilde{\mathfrak{t}}_{w}$ is dual to the cone $f^{*}(w \mathcal{C})$.

Proof. Let $\tilde{\xi} \in \tilde{\mathfrak{t}}$. Then $f^{*}(w \alpha)(\tilde{\xi}) \geq 0$ for all $\alpha \in R_{+}$if and only if $\alpha\left(w^{-1} \tilde{\xi}\right) \geq 0$ for all $\alpha \in R_{+}$. This is equivalent to $w^{-1} \tilde{\xi} \in \mathfrak{t}_{+}$, i.e. $\tilde{\xi} \in w \mathfrak{t}_{+} \cap \tilde{\mathfrak{t}}=\tilde{\mathfrak{t}}_{w}$.

2.2.5. Definition. The compatible Weyl set $W_{\text {com }}$ is the set of all $w \in W$ such that $w \mathfrak{t}_{+}$is compatible with $\tilde{\mathfrak{t}}_{+}$.

Clearly $1 \in W_{\text {com }}$ because $\mathfrak{t}_{+}$is assumed to be compatible with $\tilde{\mathfrak{t}}_{+}$, and $\bar{W} W_{\text {com }}=$ $W_{\text {com }}$ because of Lemma 2.2.1(iii). Here are some further helpful properties.

2.2.6. Proposition. Let $w \in W_{\text {com. }}$. Then

(i) $\tilde{\mathfrak{t}}_{\bar{w} w}=\tilde{\mathfrak{t}}_{w}$ and $f^{*}(\bar{w} w \mathcal{C})=f^{*}(w \mathcal{C})$ for $\bar{w} \in \bar{W}$,

(ii) $\tilde{\mathfrak{t}}_{w} \subseteq \tilde{\mathfrak{t}}_{+}$and $\tilde{\mathcal{C}} \subseteq f^{*}(w \mathcal{C})$,

(iii) $\left(f^{*}\right)^{-1}\left(\tilde{R}_{+}\right) \cap R \subseteq w R_{+}$and $\left(f^{*}\right)^{-1}\left(\tilde{R}_{-}\right) \cap R \subseteq w R_{-}$,

(iv) $\tilde{R}_{+} \subseteq f^{*}\left(w R_{+}\right)$and $\tilde{R}_{-} \cap f^{*}\left(w R_{+}\right)=\emptyset$, 
(v) $\tilde{B}=w B w^{-1} \cap \tilde{G}$,

(vi) $f^{*}(w \mathcal{C})$ is a proper cone: $f^{*}(w \mathcal{C}) \cap-f^{*}(w \mathcal{C})=\{0\}$.

Proof. (ii) follows from Lemma 2.2.1(iii).

The first statement in (iii) is proved by setting $\mathfrak{s}=w \mathfrak{t}_{+}$and $\tilde{\mathfrak{s}}=\tilde{\mathfrak{t}}_{+}$in Lemma 2.2.1(1ii). The second statement then follows from Lemma 2.2.4

Consider $\tilde{\alpha} \in \tilde{R}_{+}$and $\alpha \in R$ such that $f^{*}(\alpha)=\tilde{\alpha}$. Pick $\tilde{\xi} \in w \mathfrak{t}_{+} \cap \tilde{\mathfrak{t}}_{+}^{\circ} ;$ then $\tilde{\xi}$ can be written as $w \xi$ with $\xi \in \mathfrak{t}_{+}$. Hence

$$
\left(w^{-1} \alpha\right)(\xi)=\alpha(w \xi)=f^{*}(\alpha)(\tilde{\xi})=\tilde{\alpha}(\tilde{\xi})>0,
$$

and therefore $w^{-1} \alpha \in R_{+}$, that is, $\alpha \in w R_{+}$. This proves that $\left(f^{*}\right)^{-1}\left(\tilde{R}_{+}\right) \cap$ $R \subseteq w R_{+}$. If $\tilde{\alpha}$ is negative, we get $\left(w^{-1} \alpha\right)(\xi)<0$ in $(2.15)$, so we see that $\left(f^{*}\right)^{-1}\left(\tilde{R}_{-}\right) \cap R \subseteq w R_{-}$. This proves (iii).

(iv) follows immediately from (iii) and Lemma 2.2.1 (ii).

The first assertion in (iv) implies that $\tilde{\mathfrak{b}} \subseteq w \mathfrak{b}$ and therefore $\tilde{B} \subseteq w B w^{-1} \cap \tilde{G}$. This also shows that $w B w^{-1} \cap \tilde{G}$ is a parabolic, and hence connected, subgroup of $\tilde{G}$, so to prove the reverse inclusion we need only show that $w \mathfrak{b} \cap \tilde{\mathfrak{g}} \subseteq \tilde{\mathfrak{b}}$. This amounts to showing that the parabolic subalgebra $w \mathfrak{b} \cap \tilde{\mathfrak{g}}$ contains no negative root spaces, which follows from the second statement in (iv). This proves (语).

Because of Lemma 2.2.4 the cone $f^{*}(w \mathcal{C})$ is proper if and only if $\operatorname{dim} \tilde{\mathfrak{t}}_{w}=\operatorname{dim} \tilde{\mathfrak{t}}$. The latter is true because $\tilde{\mathfrak{t}}_{w}=w \mathfrak{t}_{+} \cap \tilde{\mathfrak{t}}_{+}$by (iii) and $\operatorname{dim} w \mathfrak{t}_{+} \cap \tilde{\mathfrak{t}}_{+}=\operatorname{dim} \tilde{\mathfrak{t}}$ by assumption. This proves (vil).

Statement vil obviously fails for any $w \in W$ such that $\tilde{\mathfrak{t}}_{w}$ is not top-dimensional. On the other hand, (iii) $-(\mathbb{v})$ are true, with exactly the same proof, under the more general hypothesis that $\tilde{\mathfrak{t}}_{w}^{\circ} \cap \tilde{\mathfrak{t}}_{+}^{\circ}$ be nonempty. Therefore (ii)-() are necessary, but not sufficient, conditions for $w$ to be a compatible element. The proof also shows that (iii)-(ㅁ) fail when $\tilde{\mathfrak{t}}_{w}$ is not contained in $\tilde{\mathfrak{t}}_{+}$. (When $\tilde{\mathfrak{t}}_{w}$ is contained in the boundary of $\tilde{\mathfrak{t}}_{+}$, then (iii) is true but (iii)-(清) may fail, depending on $w$.)

According to (ii) the top-dimensional cones $\mathfrak{t}_{w}$ are labelled by the left quotient $\bar{W} \backslash W_{\text {com }}$. It is well-known that every coset $\bar{W} w$ contains a unique element of minimal length, called its shortest representative. A more convenient set of labels is obtained by selecting from each coset the shortest representative.

2.2.7. Definition. The relative $W e y l$ set $W_{\text {rel }}$ is the set of shortest representatives of the left quotient $\bar{W} \backslash W_{\text {com }}$. For $v \in W_{\text {rel }}$ the cone $\tilde{\mathfrak{t}}_{v}$ is called a cubicle in $\tilde{\mathfrak{t}}_{+}$.

The sets $W_{\text {com }}$ and $W_{\text {rel }}$ depend of course on the choice of $\mathfrak{t}_{+}$and $\tilde{\mathfrak{t}}_{+}$. We emphasize that in general neither $W_{\text {com }}$ nor $W_{\text {rel }}$ are subgroups of $W$. We now show that the collection of cubicles forms a well-behaved conical subdivision of $\tilde{\mathfrak{t}}_{+}$ which is in one-to-one correspondence with $W_{\text {rel }}$.

2.2.8. Proposition. (i) The chamber $\tilde{\mathfrak{t}}_{+}$is the union of the cubicles,

$$
\tilde{\mathfrak{t}}_{+}=\bigcup_{v \in W_{\text {rel }}} \tilde{\mathfrak{t}}_{v},
$$

and the intersection of any two cubicles is a face of each.

(ii) If $\tilde{\mathfrak{t}}_{v_{1}}^{\circ} \cap \tilde{\mathfrak{t}}_{v_{2}} \neq \emptyset$ for some $v_{1}$ and $v_{2} \in W_{\text {rel }}$, then $v_{1}=v_{2}$.

(iii) $W_{\text {rel }}=\{1\}$ if and only if $\tilde{\mathcal{C}}=f^{*}(\mathcal{C})$.

Proof. The inclusion $\bigcup_{v \in W_{\text {rel }}} \tilde{\mathfrak{t}}_{v} \subseteq \tilde{\mathfrak{t}}_{+}$follows from Proposition 2.2.6(ii). Because $\mathfrak{t}$ is the union of its chambers $w \mathfrak{t}_{+}$, the positive chamber $\tilde{\mathfrak{t}}_{+}$of $\tilde{\mathfrak{t}}$ is the union of all 
cones $\tilde{\mathfrak{t}}_{w}$ with $\tilde{\mathfrak{t}}_{w}^{\circ} \cap \tilde{\mathfrak{t}}_{+} \neq \emptyset$. From this finite union we can clearly delete those cones whose dimension is less than $\operatorname{dim} \tilde{\mathfrak{t}}$, which leaves only the cubicles. The fact that the intersection of two cubicles is a face of each follows from the corresponding fact for the subdivision $\bigcup_{w \in W} w \mathfrak{t}_{+}$of $\mathfrak{t}$. This proves (ii).

If $\tilde{\mathfrak{t}}_{v_{1}}^{\circ} \cap \tilde{\mathfrak{t}}_{v_{2}} \neq \emptyset$, then $\tilde{\mathfrak{t}}_{v_{1}}=\tilde{\mathfrak{t}}_{v_{2}}$ by (ii). The cone $\tilde{\mathfrak{t}}_{v_{1}}=\tilde{\mathfrak{t}}_{v_{2}}$ has an open relative interior in $\tilde{\mathfrak{t}}_{+}$and furthermore $W_{\tilde{\xi}}=W_{\overline{\mathbf{c}}}=\bar{W}$ for an open dense set of $\tilde{\xi} \in \tilde{\mathfrak{t}}_{+}^{\circ}$. This implies that there exists $\tilde{\xi}$ in $v_{1} \mathfrak{t}_{+} \cap \tilde{\mathfrak{t}}_{+}^{\circ}=v_{2} \mathfrak{t}_{+} \cap \tilde{\mathfrak{t}}_{+}^{\circ}$ such that $W_{\tilde{\xi}}=\bar{W}$. This in turn implies that the two faces $v_{1} \mathfrak{t}_{+} \cap \overline{\mathfrak{c}}$ and $v_{2} \mathfrak{t}_{+} \cap \overline{\mathfrak{c}}$ of $\mathfrak{t}$ are equal to one another. Let $w=v_{2} v_{1}^{-1}$; then $w$ maps the chamber $v_{1} \mathfrak{t}_{+}$to the chamber $v_{2} \mathfrak{t}_{+}$and therefore must fix their intersection $v_{1} \mathfrak{t}_{+} \cap \overline{\mathfrak{c}}=v_{2} \mathfrak{t}_{+} \cap \overline{\mathfrak{c}}$. But then $w$ fixes $\overline{\mathfrak{c}}$, so $w \in \bar{W}$, i.e. $v_{2} \in v_{1} \bar{W}$. As $v_{1}$ and $v_{2}$ are both shortest representatives of their cosets $\bmod \bar{W}$, we conclude that $v_{1}=v_{2}$. This proves (ii).

Dualizing the condition $\tilde{\mathcal{C}}=f^{*}(\mathcal{C})$ we obtain $\tilde{\mathfrak{t}}_{+}=\tilde{\mathfrak{t}}_{1}$. The latter condition is equivalent to the subdivision (2.16) consisting of one cubicle only. According to (iii) this means that $W_{\text {rel }}=\{1\}$. This proves (iii).

We show in Section 3.4 that the subdivision (2.16) is symmetric under the standard (duality) involution of $\tilde{\mathfrak{t}}_{+}$.

Observe that the cubicles are proper cones if and only if $\tilde{T} \cap C$ is discrete. Indeed, every cubicle $\tilde{\mathfrak{t}}_{v}$ contains the linear subspace $\tilde{\mathfrak{t}} \cap \mathfrak{c}=\operatorname{Lie}(\tilde{T} \cap C)$. On the other hand, $\mathfrak{c}$ is the largest subspace contained in $v \mathfrak{t}_{+}$for all $v$, so if $\tilde{\mathfrak{t}} \cap \mathfrak{c}=0$, then $\tilde{\mathfrak{t}}_{v}$ is a proper cone.

It is clear from Proposition 2.2.8 that by intersecting all the chambers in $\tilde{\mathfrak{t}}$ with chambers in $\mathfrak{t}$ one obtains a subdivision of the entire Cartan subalgebra $\tilde{\mathfrak{t}}$ into polyhedral cones which refines the subdivision $\tilde{\mathfrak{t}}=\bigcup_{\tilde{w} \in \tilde{W}} \tilde{w} \tilde{\mathfrak{t}}_{+}$and which, by Corollary 2.1.5, is $\widetilde{W}$-invariant.

An illustration of Propositions 2.2.6 and 2.2.8 can be found in Section 2.3. For the applications in Section 3 it would be highly desirable to find the inequalities that describe each of the cones $f^{*}(v \mathcal{C})$, or equivalently, find an explicit set of spanning vectors for each of the cubicles $\tilde{\mathfrak{t}}_{v}$. This we have not been able to do in general.

We conclude this section with some miscellaneous observations. Let $W^{\prime}$ be the set of all $w \in W$ such that $\left(f^{*}\right)^{-1}\left(\tilde{R}_{+}\right) \cap R \subseteq w R_{+}$. By Proposition 2.2.6 and the remarks following it, $W^{\prime}$ includes $W_{\text {com }}$ as well as all other $w$ such that $\tilde{\mathfrak{t}}_{w}$ contains interior points of $\tilde{\mathfrak{t}}_{+}$. Put $R^{\prime}=\bigcap_{w \in W^{\prime}} w R_{+}$and $\mathcal{C}^{\prime}=\bigcap_{w \in W^{\prime}} w \mathcal{C}$.

2.2.9. Proposition. (i) $\tilde{R}_{+} \subseteq f^{*}\left(R^{\prime}\right)$.

(ii) $\tilde{\mathcal{C}}=f^{*}\left(\mathcal{C}^{\prime}\right)$.

(iii) $\bar{S}=v S \cap \bar{R}$ for all $v \in W_{\text {rel }}$.

Proof. (ii) follows immediately from the definition of $R^{\prime}$.

For any subset $\mathfrak{S}$ of a vector space $\mathfrak{E}$ we define $\mathfrak{S}^{\vee}$ to be the cone in $\mathfrak{E}^{*}$ consisting of all functionals which are nonnegative on $\mathfrak{S}^{\text {. Then }} \mathfrak{S}_{1}^{\vee} \cap \mathfrak{S}_{2}^{\vee}=\left(\mathfrak{S}_{1} \cup \mathfrak{S}_{2}\right)^{\vee}$ for all subsets $\mathfrak{S}_{1}$ and $\mathfrak{S}_{2}$. Applying this observation to (2.16) we obtain $\tilde{\mathcal{C}}=\bigcap_{v \in W_{\text {rel }}} \tilde{\mathfrak{t}}_{v}^{v}$. By Lemma 2.2.4 this implies $\tilde{\mathcal{C}}=\bigcap_{v \in W_{\text {rel }}} f^{*}(v \mathcal{C})$, and hence $f^{*}\left(\mathcal{C}_{\text {rel }}\right) \subseteq \tilde{\mathcal{C}}$. The reverse inclusion follows from (ii). This proves (iii).

For $w \in W$ let $\overline{\mathfrak{c}}_{w}$ be the cone $w \boldsymbol{t}_{+} \cap \overline{\mathfrak{c}}$. We assert that for all $w \in W$

$$
\operatorname{dim} \overline{\mathfrak{c}}_{w}=\operatorname{dim} \overline{\mathfrak{c}} \Longleftrightarrow w S \cap \bar{R} \text { is a base of } \bar{R} .
$$


Indeed, if $\operatorname{dim} \overline{\mathfrak{c}}_{w}=\operatorname{dim} \overline{\mathfrak{c}}$, then $\overline{\mathfrak{c}}$ is the linear span of $\overline{\mathfrak{c}}_{w}$. Furthermore, $w R_{+} \cap \bar{R}$ is clearly a set of positive roots for $\bar{R}$. Let $\gamma \in w R_{+} \cap \bar{R}$ and write $\gamma=w \beta$ with $\beta \in R_{+}$. Let $\alpha \in S$ be a simple root that contributes to $\beta$. Then $\alpha \geq 0$ on $\mathfrak{t}_{+}$, so $w \alpha \geq 0$ on $\overline{\mathfrak{c}}_{w}$. On the other hand, $\gamma=w \beta \in \bar{R}$, so $w \beta$ vanishes on $\overline{\mathfrak{c}}$. This is only possible if $w \alpha$ vanishes on $\overline{\mathfrak{c}}_{w}$. Hence $w \alpha$ vanishes on $\overline{\mathfrak{c}}$ and therefore $w \alpha \in \bar{R}$. We conclude that $\gamma$ is a linear combination with nonnegative coefficients of elements in $w S \cap \bar{R}$, so this set forms a base of $\bar{R}$. Conversely, if $w S \cap \bar{R}$ is a base of $\bar{R}$, then $\overline{\mathfrak{c}}$, resp. $\overline{\mathfrak{c}}_{w}$, is equal to the product $\mathfrak{c} \times \mathfrak{d}$, where $\mathfrak{c}$ is the centre of $\mathfrak{k}$ and $\mathfrak{d}$ is the linear, resp. positive, span of all fundamental coweights that are perpendicular to $w S \cap \bar{R}$. Hence $\operatorname{dim} \overline{\mathfrak{c}}_{w}=\operatorname{dim} \overline{\mathfrak{c}}$, which finishes the proof of (2.17).

Now observe that $\operatorname{dim} \overline{\mathfrak{c}}_{v}=\operatorname{dim} \overline{\mathfrak{c}}$ because $v$ is compatible. We infer from (2.17) that $v S \cap \bar{R}$ is a base of $\bar{R}$. Therefore $v S \cap \bar{R}=\bar{v}^{-1} \bar{S}$ for some $\bar{v} \in \bar{W}$, or equivalently, $\bar{w} v S \cap \bar{R}=\bar{S}$. This implies that

$$
\bar{w} v R_{+} \cap \bar{R}=\bar{R}_{+} .
$$

Take any $\alpha \in R_{+}$. If $v \alpha \in \bar{R}$, then $\bar{w} v \alpha \in \bar{R}_{+}$because of (2.18). If $v \alpha$ is not in $\bar{R}$, then $v \alpha \in R_{+}$if and only if $\bar{w} v \alpha \in R_{+}$, because $\bar{w} \in \bar{W}$ permutes the elements of $R_{+} \backslash \bar{R}_{+}$. This shows that the length of $v$, which is equal to the cardinality of $v R_{+} \cap R_{-}$, is greater than or equal to the length of $\bar{w} v$. But then $\bar{w}=1$, because $v$ is the shortest element in $\bar{W} v$. The result is that $v S \cap \bar{R}=\bar{S}$.

The condition (iii) is not sufficient for $v$ to be in $W_{\text {rel }}$ for the following reason. The proof shows that (iii) holds for any $v \in W$ which is the shortest $\bar{W}$-representative of a $w \in W$ such that $\operatorname{dim} \overline{\mathfrak{c}}_{w}=\operatorname{dim} \overline{\mathfrak{c}}$. Let us take $w$ such that $\tilde{\mathfrak{t}}_{w}$ is compatible with $\tilde{w} \tilde{\mathfrak{t}}_{+}$, where $\tilde{w}$ is an arbitrary element of $\widetilde{W}$. Then $\operatorname{dim} \overline{\mathfrak{c}}_{w}=\operatorname{dim} \overline{\mathfrak{c}}$, but if $\tilde{w} \neq 1$, then $w \notin W_{\text {rel }}$, and therefore the shortest representative of $w$ is not in $W_{\text {rel }}$.

2.3. Plethysms. Interesting examples are provided by representation theory. A unitary representation of an arbitrary compact connected group $\tilde{K}$ on a finitedimensional Hermitian vector space $V$ is nothing but a homomorphism $f: \tilde{K} \rightarrow K$, where $K$ is the unitary group $\mathbf{U}(V)$. Note that every nontrivial representation has finite kernel if $\tilde{K}$ is simple. We begin by stating some generalities concerning this class of examples and then work out a simple case in more detail.

Let $V=\bigoplus_{\mu \in \tilde{\Lambda}^{*}} V^{\mu}$ be the weight-space decomposition of $V$ and let $n(\mu)$ denote the multiplicity of a weight $\mu \in \tilde{\Lambda}^{*}$. Then $\sum n(\mu)=n=\operatorname{dim} V$. The centralizer $\bar{K}=\mathcal{Z}_{K}(\tilde{T})$ and its centre $\bar{C}$ are given by

$$
\bar{K}=\prod_{\mu} \mathbf{U}\left(V^{\mu}\right), \quad \bar{C}=\prod_{\mu} C^{\mu}
$$

where $C^{\mu}$ denotes the one-dimensional centre of $\mathbf{U}\left(V^{\mu}\right)$. Let us choose a maximal torus $T$ of $\mathbf{U}(V)$ which contains $f(\tilde{T})$. This boils down to a choice of (unordered) bases $\mathcal{B}^{\mu}=\left\{e_{1}^{\mu}, e_{2}^{\mu}, \ldots, e_{n(\mu)}^{\mu}\right\}$ of the weight spaces $V^{\mu}$. Let $\mathcal{B}$ be the basis

$$
\mathcal{B}=\coprod_{\mu} \mathcal{B}^{\mu}
$$

of $V$. Then the Lie algebra $\mathfrak{t}$ of $T$ can be identified with the real span of $\mathcal{B}$ by sending $h \in \mathfrak{t}$ to the vector

$$
\sum_{(\mu, k)} h\left(e_{k}^{\mu}\right) e_{k}^{\mu}
$$


The Weyl group $W=S_{n}$ is the group of permutations of $\mathcal{B} ; \mathcal{N}_{W}(\bar{C})$ consists of those permutations that preserve the decomposition (2.19); and $\bar{W}=\mathcal{Z}_{W}(\bar{C})=\prod_{\mu} S_{n(\mu)}$ consists of those permutations that map each $\mathcal{B}^{\mu}$ into itself. Let us identify $\mathfrak{t}$ with $\mathfrak{t}^{*}$ by means of the standard inner product on $\mathfrak{t}$ determined by the basis $\mathcal{B}$. Then the root system $R=R^{\vee}$ is the set of all vectors of the form $e_{k}^{\mu}-e_{l}^{\nu}$, where $(\mu, k) \neq(\nu, l)$, and the subsystem $\bar{R}$ consists of the vectors $e_{k}^{\mu}-e_{l}^{\mu}$.

Observe that $\bar{R} \neq \emptyset$ and $\bar{W} \neq\{1\}$ as soon as $V$ has a weight of multiplicity greater than 1. For any such representation $V$ all points in $f(\tilde{K})$ are singular considered as points in $\mathbf{U}(V)$.

The matrix of $f_{*}$ (relative to the coroots in $\tilde{\mathfrak{t}}$ and the basis $\mathcal{B}$ in $\mathfrak{t}$ ) and the set of projected roots $f^{*}(R) \subseteq \tilde{\mathfrak{t}}^{*}$ can be read off easily from the weight diagram of $V$.

2.3.1. Lemma. Put $e^{\mu}=\sum_{i=1}^{n(\mu)} e_{k}^{\mu}$ for all weights $\mu$ of $V$. Then the inclusion $f_{*}: \tilde{\mathfrak{t}} \hookrightarrow \overline{\mathfrak{c}} \hookrightarrow \mathfrak{t}$ is given by

$$
f_{*}(\tilde{h})=\sum_{\mu} \mu(\tilde{h}) e^{\mu} .
$$

Hence $f^{*}\left(e_{k}^{\mu}\right)=\mu$ and $f^{*}\left(e_{k}^{\mu}-e_{l}^{\nu}\right)=\mu-\nu$ for all $(\mu, k)$ and $(\nu, l)$.

Proof. For all $(\mu, k)$ we have $f_{*}(\tilde{h})\left(e_{k}^{\mu}\right)=\mu(\tilde{h}) e_{k}^{\mu}$ because $e_{k}^{\mu}$ has weight $\mu$. Therefore, according to the identification (2.20), $f_{*}(\tilde{h})$ is equal to $\sum_{\mu} \mu(\tilde{h}) e^{\mu}$.

Dually, we have

$$
\left\langle f^{*}\left(e_{k}^{\mu}\right), \tilde{h}\right\rangle=\left\langle e_{k}^{\mu}, f_{*}(\tilde{h})\right\rangle=\sum_{\nu} \nu(\tilde{h})\left\langle e_{k}^{\mu}, e^{\nu}\right\rangle=\mu(\tilde{h}),
$$

so $f^{*}\left(e_{k}^{\mu}\right)=\mu$.

According to the discussion following Definition 2.2.2 a compatible pair of chambers $\tilde{\mathfrak{t}}_{+}$and $\mathfrak{t}_{+}$is specified by a set of positive roots in $\bar{R}$, i.e. an ordering of each of the bases $\mathcal{B}^{\mu}$, and by a set of positive roots in $\tilde{R}$, which implies an ordering $\preccurlyeq$ on the weight lattice $\tilde{\Lambda}^{*}$. This gives rise to an ordering on the set of pairs $(\mu, k)$ defined by $(\mu, k) \geq(\nu, l)$ if $\mu \succcurlyeq \nu$ and $(\mu, k) \geq(\mu, l)$ if $k \geq l$. In terms of this ordering $R_{+}$is given by the set of all $e_{k}^{\mu}-e_{l}^{\nu}$ with $(\mu, k) \geq(\nu, l)$.

2.3.2. Example. Take $\tilde{K}=\mathbf{S U}(3)$ and consider the unitary irreducible representation $\tilde{V}_{\tilde{\lambda}}$ with highest weight $\tilde{\lambda}=2 \tilde{\pi}_{1}+\tilde{\pi}_{2}$, where $\tilde{\pi}_{1}$ and $\tilde{\pi}_{2}$ are the fundamental weights of $\tilde{K}$. Then $\operatorname{dim} \tilde{V}_{\tilde{\lambda}}=15$, so this representation defines an embedding $f: \tilde{K} \rightarrow K$, where $K=\mathbf{U}(15)$. We number the weights as in Figure 1 This ordering specifies a positive chamber $\mathfrak{t}_{+}$in $K$ which is compatible with the (standard) positive chamber $\tilde{\mathfrak{t}}_{+}$in $\tilde{K}$. We denote the corresponding bases of the root systems $R$ and $\tilde{R}$ by $S$ and $\tilde{S}$, respectively.

The projected roots are easy to compute from Lemma 2.3.1 and by Lemma 2.2 .4 the cubicles in $\tilde{\mathfrak{t}}_{+}$are then determined by the hyperplanes perpendicular to the $f^{*}(\alpha)$. The matrix of $f^{*}$ relative to the bases of fundamental weights in $\mathbf{S U}(15)$ and $\mathbf{S U}(3)$ is

$$
\left(\begin{array}{llllllllllllll}
2 & 5 & 5 & 6 & 7 & 5 & 7 & 6 & 5 & 5 & 5 & 2 & 3 & 1 \\
1 & 0 & 2 & 2 & 2 & 5 & 3 & 4 & 5 & 4 & 3 & 5 & 2 & 2
\end{array}\right) .
$$

The projected roots and the cubicles are shown in Figure 2, where we have used the trace form to identify $\tilde{\mathfrak{t}}$ with its dual. The arrows, with multiplicities, indicate the images of the 105 positive roots. If an arrow occurs as the image of one of 


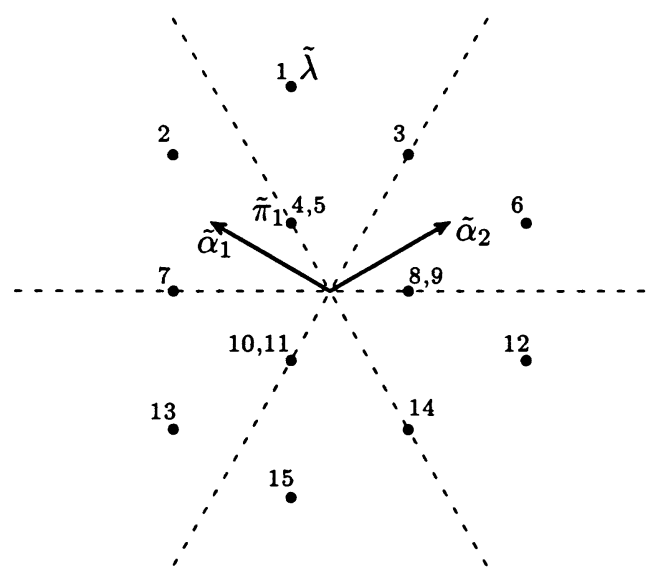

FiguRE 1. Numbering of weights for SU(3)-module with highest weight $\tilde{\lambda}=2 \tilde{\pi}_{1}+\tilde{\pi}_{2}$

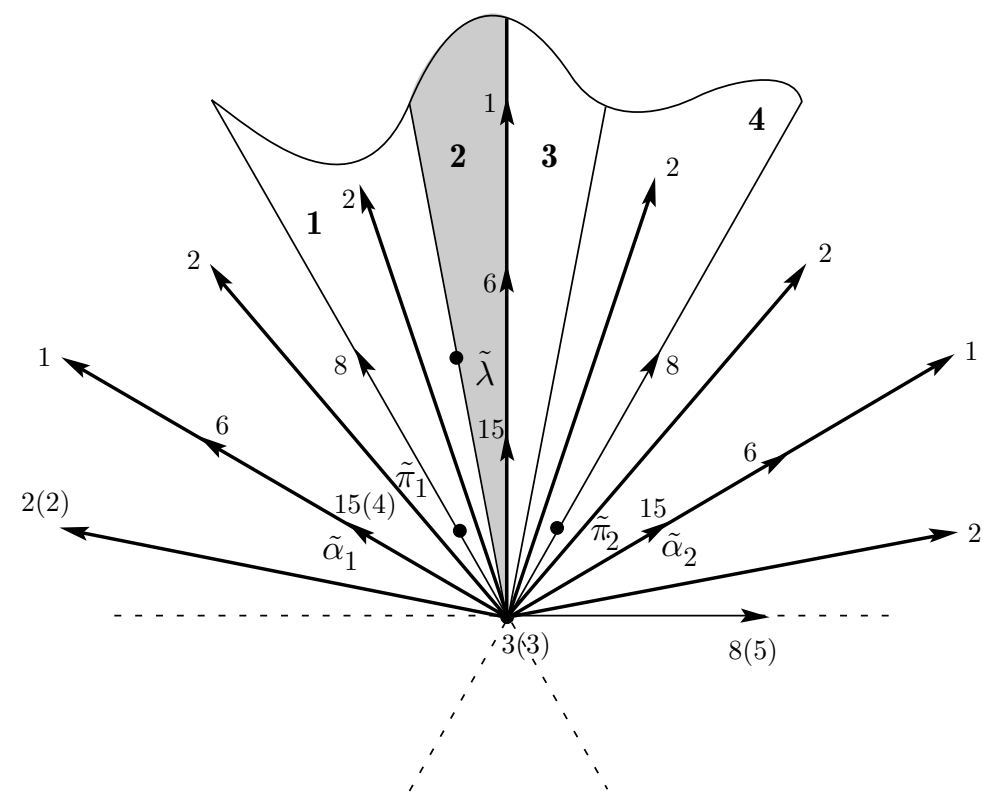

Figure 2. Peacock's tail with 105 feathers. Projected positive roots $f^{*}\left(R_{+}\right)$and cubicles $\tilde{\mathfrak{t}}_{v}$ for embedding $\mathbf{S U}(3) \rightarrow \mathbf{U}(15)$ defined by dominant weight $\tilde{\lambda}=2 \tilde{\pi}_{1}+\tilde{\pi}_{2}$

the 14 simple roots, the number of times it so occurs is written in parentheses. Three positive roots are mapped to 0 , namely $\alpha_{4}, \alpha_{8}$ and $\alpha_{10}$, so that $\bar{S}=\bar{R}_{+}=$ $\left\{\alpha_{4}, \alpha_{8}, \alpha_{10}\right\}$ and $\operatorname{dim} \bar{C}=14-3=11$.

The centralizer $\bar{K}=\mathcal{Z}_{K}(\tilde{T})=\mathcal{Z}_{K}(\bar{C})$ is a group of block-diagonal matrices with nine $1 \times 1$-blocks and three $2 \times 2$-blocks and hence is isomorphic to $\mathbf{U}(1)^{9} \times \mathbf{U}(2)^{3}$. Its Weyl group is $\bar{W}=(\mathbb{Z} / 2 \mathbb{Z})^{3}$; it is generated by the simple reflections $s_{4}, s_{8}$ and 
$s_{10}$, and acts by permuting rows and columns of each of the $2 \times 2$-blocks. The group $\mathcal{N}_{W}(\bar{C})$ is isomorphic to $S_{9} \times(\mathbb{Z} / 2 \mathbb{Z})^{3} \rtimes S_{3}$. The copy of $S_{9}$ acts on $\bar{K}$ and its centre $\bar{C}$ by permuting the $1 \times 1$-blocks, the copy of $S_{3}$ permutes the $2 \times 2$-blocks, and the copy of $(\mathbb{Z} / 2 \mathbb{Z})^{3}$ permutes the rows and columns of the individual $2 \times 2$-blocks. The group $\mathcal{N}_{W}(\bar{S})$ is isomorphic to $S_{9} \times S_{3}$. It consists of all permutations $w$ in $W \cong S_{15}$ that map the sets $\{1,2,3,6,7,12,13,14,15\}$ and $\{4,8,10\}$ into themselves and satisfy $w(i)=i+1$ for $i=4,8$, or 10 .

The homomorphism $j: \widetilde{W} \rightarrow \mathcal{N}_{W}(\bar{S})$ is found as follows. By Theorem 2.1.4 for each $\tilde{w} \in \tilde{W}$ the image of $\tilde{w}$ under $j$ is the unique $w \in \mathcal{N}_{W}(\bar{S})$ such that $f_{*}(\tilde{w} \tilde{\xi})=w f_{*}(\tilde{\xi})$ for all $\tilde{\xi} \in \tilde{\mathfrak{t}}$. In fact, in this condition it suffices to take a single $\tilde{\xi}$ in the interior of one of the cones $\tilde{\mathfrak{t}}_{v}$, e.g. $\tilde{\xi}=6 \tilde{\alpha}_{1}^{\vee}+5 \tilde{\alpha}_{2}^{\vee} \in \tilde{\mathfrak{t}}_{1}$. An easy calculation now yields that on generators $j$ is given by

$$
\begin{aligned}
& j\left(\tilde{s}_{1}\right)=\left(\begin{array}{ll}
1 & 6
\end{array}\right)(212)(48)(59)(714)(1315), \\
& j\left(\tilde{s}_{2}\right)=(12)(3 \quad 7)(613)(810)(911)(1215) \text {. }
\end{aligned}
$$

The other seven natural embeddings of $\widetilde{W}$ into $W$ are obtained by conjugating $j$ with any of the transpositions (4 5), (8 9), (10 11), or a product thereof.

The relative Weyl set consists of the four elements

$$
\begin{aligned}
& v_{1}=\left(\begin{array}{l}
6 \\
6
\end{array}\right)(1213), \quad v_{2}=1, \quad v_{3}=(23)(465)(789)(101211)(1314), \\
& v_{4}=(23654)(789121110)(1314) \text {, }
\end{aligned}
$$

which have length 2, 0, 8 and 10, respectively. The cubicles are labelled accordingly in Figure 2, the cubicle $\tilde{\mathfrak{t}}_{1}=\mathfrak{t}_{+} \cap \tilde{\mathfrak{t}}$ is shown in dark grey. We determined $W_{\text {rel }}$ by picking elements in the interior of each cubicle, namely $\tilde{\xi}_{1}=3 \tilde{\alpha}_{1}^{\vee}+2 \tilde{\alpha}_{2}^{\vee}, \tilde{\xi}_{2}=$ $6 \tilde{\alpha}_{1}^{\vee}+5 \tilde{\alpha}_{2}^{\vee}, \tilde{\xi}_{3}=5 \tilde{\alpha}_{1}^{\vee}+6 \tilde{\alpha}_{2}^{\vee}$, and $\tilde{\xi}_{4}=2 \tilde{\alpha}_{1}^{\vee}+3 \tilde{\alpha}_{2}^{\vee}$, and reflecting them into the positive chamber $\mathfrak{t}_{+}$.

This example disabused us of some overoptimistic notions we had entertained. For instance, the subgroup $f(\mathbf{S U}(3))$ consists of highly singular points in $\mathbf{U}(15)$. Moreover, the small Cartan $\tilde{\mathfrak{t}}$ has quite high codimension in $\overline{\mathfrak{c}}$. Notice also that the cones $\tilde{\mathfrak{t}}_{v}$ come in two different shapes. Furthermore, $\tilde{\alpha}_{2}$ is not in the image of $S$, so $\tilde{S}$ is not a subset of $f^{*}(S)$, although $\tilde{R}_{+}$is a subset of $f^{*}\left(R_{+}\right)$by Lemma 2.2.1 Lastly, the relative Weyl elements do not preserve the root system $\bar{R}$, so $W_{\text {rel }}$ is not a subset of $\mathcal{N}_{W}(\bar{C})$.

\section{MAin Results}

As before, let $\tilde{K}$ and $K$ be compact connected Lie groups and let $f: \tilde{K} \rightarrow K$ be a homomorphism with finite kernel. In Section 3.1 we state our main result, a complete set of inequalities for the moment cone of the cotangent bundle $T^{*} K$, considered as a $\tilde{K} \times K$-manifold. We deduce from this complete sets of inequalities for the moment cone of $T^{*}(\tilde{K} \backslash K)$ (considered as a $K$-manifold) and for the moment polytope of every coadjoint orbit of $K$ (considered as a $\tilde{K}$-manifold). As explained in the introduction, this is equivalent to an asymptotic result on the decomposition of an irreducible $K$-module into irreducible $\tilde{K}$-modules. This and other corollaries are stated in Section 3.2. The inequalities we obtain are in general highly overdetermined. In Section 3.3 we explain how to prune them to a more manageable set of inequalities. We defer the proofs to Section 4. Finally, in Section 3.4 we discuss the "self-duality" of the moment cone of $T^{*} K$ and explain it in terms of the action of the longest elements of $W$ and $\widetilde{W}$. 
3.1. Schubert cycles and the moment cone. Recall that $T$ and $\tilde{T}$ denote maximal tori of $K$ and $\tilde{K}$, respectively, such that $\tilde{T}=T \cap \tilde{K}$. Let $R \subseteq \mathfrak{t}^{*}$ and $\tilde{R} \subseteq \tilde{\mathfrak{t}}^{*}$ be the respective root systems and $W$ and $\widetilde{W}$ the associated Weyl groups. Choose bases (sets of simple roots) $S$ in $R$ and $\tilde{S}$ in $\tilde{R}$ such that the corresponding (closed) positive Weyl chambers $\mathfrak{t}_{+} \subseteq \mathfrak{t}$ and $\tilde{\mathfrak{t}}_{+} \subseteq \tilde{\mathfrak{t}}$ are compatible in the sense of Definition 2.2.2. Denote the corresponding dual Weyl chambers in $\mathfrak{t}^{*}$ and $\tilde{\mathfrak{t}}^{*}$ by $\mathfrak{t}_{+}^{*}$ and $\tilde{\mathfrak{t}}_{+}^{*}$, respectively. Consider the symplectic manifold $T^{*} K$ on which $\tilde{K}$ acts by left multiplication and $K$ by right multiplication. Let us identify $T^{*} K$ with $K \times \mathfrak{k}^{*}$ by means of left translations; then the action of $\tilde{K} \times K$ is given by

$$
(\tilde{g}, g) \cdot(k, \xi)=\left(f(\tilde{g}) k^{-1}, g \xi\right) .
$$

With respect to the standard symplectic structure on $T^{*} K$ this action has a moment $\operatorname{map} \Phi=\Phi_{\tilde{K}} \times \Phi_{K}: T^{*} K \rightarrow \tilde{\mathfrak{k}}^{*} \times \mathfrak{k}^{*}$ given by

$$
\Phi_{\tilde{K}}(k, \xi)=f^{*}(k \xi), \quad \Phi_{K}(k, \xi)=-\xi .
$$

According to [22, Theorem 7.6] the set

$$
\Delta\left(T^{*} K\right)=\Phi\left(T^{*} K\right) \cap\left(\tilde{\mathfrak{t}}_{+}^{*} \times \mathfrak{t}_{+}^{*}\right)
$$

is a rational convex polyhedral cone, called the moment cone of $T^{*} K$. Our main result is a set of inequalities describing this cone.

3.1.1. Theorem. Let $(\tilde{\lambda}, \lambda) \in \tilde{\mathfrak{t}}_{+}^{*} \times \mathfrak{t}_{+}^{*}$. Then $(\tilde{\lambda}, \lambda) \in \Delta\left(T^{*} K\right)$ if and only if

$$
\tilde{w}^{-1} \tilde{\lambda} \in f^{*}\left(-w^{-1} \lambda+v \mathcal{C}\right)
$$

for all triples $(\tilde{w}, w, v) \in \widetilde{W} \times W \times W_{\text {rel }}$ such that

$$
\phi^{*}\left(v \sigma_{w v}\right)\left(\tilde{c}_{\tilde{w}_{0} \tilde{w}}\right) \neq 0 .
$$

The proof is in Section4. First we explain the statement and point out a number of consequences. Recall that $\mathcal{C} \subseteq \mathfrak{t}^{*}$ denotes the root cone, i.e. the cone spanned by the positive roots $R_{+}$. The relative Weyl set $W_{\text {rel }}$ is the subset (not subgroup) of the Weyl group $W$ defined in 2.2.7. For each $w \in W$ and $v \in W_{\text {rel }}$ the set $f^{*}\left(-w^{-1} \lambda+v \mathcal{C}\right)$ is a polyhedral cone in $\tilde{\mathfrak{t}}^{*}$ with apex $f^{*}\left(-w^{-1} \lambda\right)$, and so (3.2) represents a finite number of linear inequalities. For instance, if $\tilde{\mathfrak{t}}_{+}$is contained in $\mathfrak{t}_{+}$, then by Proposition 2.2.8, $W_{\text {rel }}=\{1\}$ and $f^{*}(\mathcal{C})=\tilde{\mathcal{C}}$, the root cone of $\tilde{K}$.

The condition (3.3) is of a homological nature. Let $X=K / T$ be the flag variety of $K$. The Weyl group $W$ acts on the homogeneous space $X$ by right multiplication, $w(k T)=k w^{-1} T$. (We denote a Weyl group element and any of its representatives in $\mathcal{N}_{K}(T)$ by the same letter as long as the formulas do not depend on the choice of the representative.) The induced action on a homology or cohomology class $c$ is written as $w c$.

Recall that the homology of $X$ is torsion-free and has a basis given by the Schubert classes $c_{w} \in H_{2 l(w)}(X, \mathbb{Z})$, where $w$ ranges over $W$ and $l(w)$ is the length of $w$. (The definition is reviewed in Appendix A ) If $w_{0}$ is the longest Weyl group element, then $c_{w_{0}}$ is equal to the fundamental class $[X]$. The homology being torsion-free, we can identify the cohomology with the dual abelian group $\operatorname{Hom}_{\mathbb{Z}}(H \cdot(X, \mathbb{Z}), \mathbb{Z})$. The cohomology class $\sigma_{w} \in H^{2 l(w)}(X, \mathbb{Z})$ is defined by $\sigma_{w}(c)=c \cdot c_{w_{0} w}$ for $c$ in $H_{2 l(w)}(X, \mathbb{Z})$ and $\sigma_{w}(c)=0$ if $c$ has degree different from $2 l(w)$. Recall that if $l(w)+l\left(w^{\prime}\right) \leq l\left(w_{0}\right)$, then

$$
c_{w} \cdot c_{w^{\prime}}=\delta_{w, w_{0} w^{\prime}} c_{1}
$$


where the dot denotes the intersection product. This implies that the basis $\left\{\sigma_{w} \mid\right.$ $w \in W\}$ of $H^{\bullet}(X, \mathbb{Z})$ is dual to the basis $\left\{c_{w} \mid w \in W\right\}$ of $H_{\bullet}(X, \mathbb{Z})$.

Finally, the map $\phi$ is the embedding of the flag variety $\tilde{X}=\tilde{K} / \tilde{T}$ into the flag variety $X=K / T$ which is induced by the map $f: \tilde{K} \rightarrow K$. It is injective even if $f$ is not.

The Schubert bases depend on the choice of the dominant chambers. However, it is not hard to work out the formula for a change of basis (see e.g. Remark A.1.1) and to show that a different choice of compatible chambers $\tilde{\mathfrak{t}}_{+}$and $\mathfrak{t}_{+}$leads up to a relabelling to the same set of inequalities.

Theorem 3.1 .1 makes the inequalities of the moment cone computable in practice once we can

(i) determine the relative Weyl set $W_{\text {rel }}$,

(ii) write down inequalities for the codimension-one faces of the cones $f^{*}(v \mathcal{C})$, in other words, determine the rays that span their dual cones,

(iii) write the action of the elements of $W_{\text {rel }}$ on the homology in terms of the Schubert basis,

(iv) find the nonzero entries of the matrix of $\phi^{*}$ with respect to the Schubert bases.

Unfortunately, we do not know of a general solution to these combinatorial problems except the third. (Formulas for the Weyl group action on the homology in terms of the Schubert basis were given by Bernstein et al. [3] and Demazure [6]. These are reviewed in Appendix A In many interesting examples, however, they can be solved explicitly. Some properties that can be helpful in computing $W_{\text {rel }}$ and the cones $f^{*}(v \mathcal{C})$ are discussed in Section 2.2. Problem (iv) is discussed in Appendix $\mathrm{A}$

3.2. Alternative formulations and corollaries. Let $\lambda \in \mathfrak{t}_{+}^{*}$ and consider the coadjoint orbit $\mathcal{O}_{\lambda}=K \lambda$ through $\lambda$. It follows from (3.1) that the symplectic quotient $\Phi_{K}^{-1}\left(\mathcal{O}_{\lambda}\right) / K$ of $T^{*} K$ with respect to the right $K$-action is isomorphic, as a Hamiltonian $\tilde{K}$-manifold, to the dual orbit $\mathcal{O}_{-w_{0} \lambda}$. Consequently the moment polytope $\Delta\left(\mathcal{O}_{-w_{0} \lambda}\right)$ of $\mathcal{O}_{-w_{0} \lambda}$ with respect to the $\tilde{K}$-action is equal to the horizontal slice

$$
\Delta\left(\mathcal{O}_{-w_{0} \lambda}\right)=\Delta\left(T^{*} K\right) \cap\left(\tilde{\mathfrak{t}}^{*} \times\{\lambda\}\right)
$$

of the moment cone $\Delta\left(T^{*} K\right)$. Furthermore, it is evident that $\tilde{\lambda} \in \Delta\left(\mathcal{O}_{\lambda}\right)$ if and only if $-\tilde{w}_{0} \tilde{\lambda} \in \Delta\left(\mathcal{O}_{-w_{0} \lambda}\right)$. Thus, after substituting $\tilde{w} \rightarrow \tilde{w}_{0} w$, we see that Theorem 3.1.1 is equivalent to the following statement.

3.2.1. Theorem. Let $(\tilde{\lambda}, \lambda) \in \tilde{\mathfrak{t}}_{+}^{*} \times \mathfrak{t}_{+}^{*}$. Then $\tilde{\lambda} \in \Delta\left(\mathcal{O}_{\lambda}\right)$ if and only if

$$
\tilde{w}^{-1} \tilde{\lambda} \in f^{*}\left(w^{-1} \lambda-v \mathcal{C}\right)
$$

for all triples $(\tilde{w}, w, v) \in \widetilde{W} \times W \times W_{\text {rel }}$ such that $\phi^{*}\left(v \sigma_{w v}\right)\left(\tilde{c}_{\tilde{w}}\right) \neq 0$.

Because of the equivalence of problems (ii) and (ii') discussed in the introduction, Theorem 3.2 .1 is tantamount to an asymptotic statement about irreducible representations. Let $\Lambda=\operatorname{ker}\left(\left.\exp \right|_{\mathfrak{t}}\right)$ be the integral lattice in $\mathfrak{t}, \Lambda^{*}=\operatorname{Hom}_{\mathbb{Z}}(\Lambda, \mathbb{Z})$ the weight lattice and $\Lambda_{+}^{*}=\Lambda^{*} \cap \mathfrak{t}_{+}^{*}$ the monoid of dominant weights. For every dominant weight $\lambda$ let $V_{\lambda}$ denote the irreducible $K$-module with highest weight $\lambda$. 
3.2.2. Theorem. Let $(\tilde{\lambda}, \lambda) \in \tilde{\Lambda}_{+}^{*} \times \Lambda_{+}^{*}$. Then there exists a positive integer $n$ such that $\tilde{V}_{n \tilde{\lambda}}$ occurs in $V_{n \lambda}$ if and only if (3.5) holds for all $(\tilde{w}, w, v) \in \widetilde{W} \times W \times W_{\text {rel }}$ such that $\phi^{*}\left(v \sigma_{w v}\right)\left(\tilde{c}_{\tilde{w}}\right) \neq 0$.

Similarly, it follows from (3.1) that the symplectic quotient $\tilde{K} \backslash \Phi_{\tilde{K}}^{-1}(0)$ of $T^{*} K$ with respect to the left $\tilde{K}$-action is symplectomorphic to the cotangent bundle of the homogeneous space $\tilde{K} \backslash K$. Hence the moment cone of $T^{*}(\tilde{K} \backslash K)$ with respect to the residual $K$-action is equal to the "vertical slice"

$$
\Delta\left(T^{*}(\tilde{K} \backslash K)\right)=\Delta\left(T^{*} K\right) \cap\left(\{0\} \times \mathfrak{t}^{*}\right)
$$

of the moment cone $\Delta\left(T^{*} K\right)$. The following result is now immediate from Theorem 3.1 .1 .

3.2.3. Theorem. Let $\lambda \in \mathfrak{t}_{+}^{*}$. Then $\lambda \in \Delta\left(T^{*}(\tilde{K} \backslash K)\right)$ if and only if

$$
0 \in f^{*}\left(w^{-1} \lambda-v \mathcal{C}\right)
$$

for all pairs $(w, v) \in W \times W_{\text {rel }}$ such that $\phi^{*}\left(v \sigma_{w v}\right) \neq 0$.

Like Theorem 3.2.1 this has a representation-theoretic counterpart.

3.2.4. Theorem. Let $\lambda \in \Lambda_{+}^{*}$. Then there exists $n>0$ such that $V_{n \lambda}$ contains a $\tilde{K}$-invariant vector if and only if (3.6) holds for all $(w, v) \in W \times W_{\text {rel }}$ such that $\phi^{*}\left(v \sigma_{w v}\right) \neq 0$.

Although we have presented Theorems 3.2 .3 and 3.2 .4 as corollaries of Theorem 3.1.1. they are actually equivalent to it and play a part in the proof; see Section 4.3 .

Suppose now that $\tilde{V}_{\tilde{\lambda}}$ occurs in $V_{\lambda}$. Then the weights in $\tilde{V}_{\tilde{\lambda}}$ are a subset of the weights of $V_{\lambda}$ (with respect to the torus $\tilde{T}$ ). The latter are of the form $f^{*}(\lambda-\mu)$, where $\mu$ is a combination of positive roots of $K$ with nonnegative integral coefficients. In particular, $\tilde{\lambda}$ itself is of this form. From Theorem 3.2 .2 we thus obtain the following necessary condition for $V_{\lambda}$ to contain $\tilde{V}_{\tilde{\lambda}}$.

3.2.5. Theorem. Let $(\tilde{\lambda}, \lambda) \in \tilde{\Lambda}_{+}^{*} \times \Lambda_{+}^{*}$. If $\tilde{V}_{\tilde{\lambda}}$ occurs in $V_{\lambda}$, then

$$
\tilde{w}^{-1} \tilde{\lambda} \in f^{*}\left(w^{-1} \lambda-v \mathcal{C}_{\mathbb{Z}}\right)
$$

for all triples $(\tilde{w}, w, v) \in \widetilde{W} \times W \times W_{\text {rel }}$ such that $\phi^{*}\left(v \sigma_{w v}\right)\left(\tilde{c}_{\tilde{w}}\right) \neq 0$.

Here we have written $\mathcal{C}_{\mathbb{Z}}$ for the monoid generated by $R_{+}$. An interesting question is for what pairs $K, \tilde{K}$ this necessary condition is sufficient.

3.2.6. Example. The answer to this question is clearly affirmative when $\tilde{K}=T$, the maximal torus of $K$. The same is true for the diagonal embedding of $\mathbf{U}(n)$ into $\mathbf{U}(n) \times \mathbf{U}(n)$, as was recently shown by Knutson and Tao [16. However, it was pointed out to us by Knop that the answer is negative for the diagonal embedding $\mathbf{G}_{2} \rightarrow \mathbf{G}_{2} \times \mathbf{G}_{2}$ : the module $V_{03}$ is contained in $V_{30} \otimes V_{03}$, but $V_{01}$ is not contained in $V_{10} \otimes V_{01}$. Here $V_{k l}$ is the irreducible module with highest weight $k \pi_{1}+l \pi_{2}, \pi_{1}$ and $\pi_{2}$ being the fundamental weights of $\mathbf{G}_{2}$. This can be checked using a result of Littelmann [18 §3.8]. See Brion [5] and Montagar [19] for further references and related results. 
We discuss two more reformulations of Theorem 3.1.1. First, for all $(\tilde{w}, w) \in$ $\widetilde{W} \times W$ let $\mathcal{C}_{\tilde{w}, w}$ be the cone $\bigcap_{v} f^{*}(v \mathcal{C})$, where the intersection is taken over all $v \in W_{\text {rel }}$ such that (3.3) holds (and put $\mathcal{C}_{\tilde{w}, w}=\tilde{\mathfrak{t}}^{*}$ if there is no such $v$ ). Theorem 3.1.1 is equivalent to: $(\tilde{\lambda}, \lambda) \in \Delta\left(T^{*} K\right)$ if and only if $(\tilde{\lambda}, \lambda) \in \tilde{\mathfrak{t}}_{+}^{*} \times \mathfrak{t}_{+}^{*}$ and

$$
\tilde{w}^{-1} \tilde{\lambda}+f^{*}\left(w^{-1} \lambda\right) \in \mathcal{C}_{\tilde{w}, w}
$$

for all $(\tilde{w}, w)$.

Next, let $\mathcal{P}: H^{i}(X, \mathbb{Z}) \rightarrow H_{2 l\left(w_{0}\right)-i}(X, \mathbb{Z})$ denote the Poincaré duality map, $\mathcal{P}(\sigma)=\sigma \cap[X]$. Then $\mathcal{P}\left(\sigma_{w}\right)=c_{w_{0} w}$ by (3.4). Let

$$
\phi^{!}=\tilde{\mathcal{P}} \circ \phi^{*} \circ \mathcal{P}^{-1}: H_{\bullet}(X, \mathbb{Z}) \rightarrow H_{\bullet+2\left(l\left(\tilde{w}_{0}\right)-l\left(w_{0}\right)\right)}(\tilde{X}, \mathbb{Z})
$$

be the wrong-way or Gysin homomorphism induced by $\phi$. If $l(\tilde{w})+l(w v)=l\left(\tilde{w}_{0}\right)$, then $\operatorname{deg} \phi^{*}\left(v \sigma_{w v}\right)=\operatorname{deg} \tilde{c}_{\tilde{w}_{0} \tilde{w}}$, so

$$
\begin{aligned}
\phi^{*}\left(v \sigma_{w v}\right)\left(\tilde{c}_{\tilde{w}_{0} \tilde{w}}\right) & =\tilde{\mathcal{P}}\left(\phi^{*}\left(v \sigma_{w v}\right)\right) \cdot \tilde{c}_{\tilde{w}_{0} \tilde{w}} \\
& =\phi^{!}\left(\mathcal{P}\left(v \sigma_{w v}\right)\right) \cdot \tilde{c}_{\tilde{w}_{0} \tilde{w}} \\
& =(-1)^{l(v)} \phi^{!}\left(v \mathcal{P} \sigma_{w v}\right) \cdot \tilde{c}_{\tilde{w}_{0} \tilde{w}} \\
& =(-1)^{l(v)} \phi^{!}\left(v c_{w_{0} w v}\right) \cdot \tilde{c}_{\tilde{w}_{0} \tilde{w}}
\end{aligned}
$$

Here we have used that the mapping degree of the diffeomorphism of $X$ induced by $v$ is equal to $(-1)^{l(v)}$, which follows from TheoremA.2.3. Let us say that a Schubert class $c_{w}$ is contained in a homology class $c$ if it occurs with nonzero coefficient in the expression for $c$ as a linear combination of Schubert classes. This amounts to $\sigma_{w}(c) \neq 0$, or equivalently, if $c$ is homogeneous, $c \cdot c_{w_{0} w} \neq 0$ and $\operatorname{deg} c=2 l(w)$. Then according to (3.7), condition (3.3) is equivalent to

$$
\tilde{c}_{\tilde{w}} \quad \text { is contained in } \phi !\left(v c_{w_{0} w v}\right) \text {. }
$$

This means that there exist cycles on $\tilde{X}$ and $X$ which are homologous to $\tilde{c}_{\tilde{w}}$ and $v c_{w_{0} w v}$, respectively, and which have a nontrivial transverse intersection. Applying $\tilde{\mathcal{P}}^{-1}$ to both sides yields a cohomological version of (3.8):

$$
\tilde{\sigma}_{\tilde{w}_{0} \tilde{w}} \text { is contained in } \phi^{*}\left(v \sigma_{w v}\right) .
$$

Observe also that the condition $\phi^{*}\left(v \sigma_{w v}\right)\left(\tilde{c}_{\tilde{w}}\right) \neq 0$ of Theorem 3.2 .1 is equivalent to

$$
\tilde{\sigma}_{\tilde{w}} \text { is contained in } \phi^{*}\left(v \sigma_{w v}\right) \text {. }
$$

This reformulation of Theorem 3.2.1 does not refer to Poincaré duality or the longest Weyl group elements and therefore makes sense, at least syntactically, for coadjoint orbits of certain infinite-dimensional groups.

3.3. Scalar inequalities. Many of the vector inequalities (3.2) are redundant. In fact, each of them can be replaced by a single scalar inequality, and in addition (3.3) can be reduced to a much smaller set of homological conditions. To do so we need to recall some more results from [3].

Let $\mathfrak{s}$ be a (closed) face of the Weyl chamber $\mathfrak{t}_{+}$. The centralizer of $\mathfrak{s}$ is equal to the centralizer of any point in its (relative) interior $\mathfrak{s}^{\circ}$ and is denoted by $\mathcal{Z}_{K}(\mathfrak{s})$ or $K_{\mathfrak{s}}$. It is a connected subgroup of $K$ and contains the maximal torus $T$. Its Weyl group $\mathcal{N}_{K_{\mathfrak{s}}}(T) / T$ is denoted by $W_{\mathfrak{s}}$, and the partial flag variety $K / K_{\mathfrak{s}}$ by $X_{\mathfrak{s}}$. The 
homology of $X_{\mathfrak{s}}$ has a free basis consisting of Schubert classes $c_{w}^{\mathfrak{s}} \in H_{2 l(w)}\left(X_{\mathfrak{s}}, \mathbb{Z}\right)$, where $w$ ranges over the set $W^{\mathfrak{s}}$ defined by

$$
W^{\mathfrak{s}}=\left\{w \in W \mid l\left(w w^{\prime}\right) \geq l(w) \quad \text { for all } w^{\prime} \in W_{\mathfrak{s}}\right\} .
$$

Every coset $w W_{\mathfrak{s}}$ contains a unique element in $W^{\mathfrak{s}}$, called its shortest representative. The canonical projection $X \rightarrow X_{\mathfrak{s}}$ induces a surjection $H_{\bullet}(X, \mathbb{Z}) \rightarrow H_{\bullet}\left(X_{\mathfrak{s}}, \mathbb{Z}\right)$, which maps $c_{w}$ to $c_{w}^{\mathfrak{s}}$ if $w \in W^{\mathfrak{s}}$ and to 0 if $w \notin W^{\mathfrak{s}}$. It also induces an embedding of $H^{\bullet}\left(X_{\mathfrak{s}}, \mathbb{Z}\right)$ onto the subspace of $W_{\mathfrak{s}}$-invariants in $H^{\bullet}(X, \mathbb{Z})$. A basis of $H^{\bullet}(X, \mathbb{Z})^{W_{\mathfrak{s}}}$ is given by the Schubert cocycles $\left\{\sigma_{w} \mid w \in W^{\mathfrak{s}}\right\}$. Via the identification $H^{\bullet}\left(X_{\mathfrak{s}}, \mathbb{Z}\right) \cong H^{\bullet}(X, \mathbb{Z})^{W_{\mathfrak{s}}}$ this basis is dual to $\left\{c_{w}^{\mathfrak{s}} \mid w \in W^{\mathfrak{s}}\right\}$. The Poincaré dual of $\sigma_{w}$ is $c_{u}^{\mathfrak{s}}$, where $u$ is the shortest representative of $w_{0} w W_{\mathfrak{s}}$.

Consider any point $\tilde{\chi}$ in $\tilde{\mathfrak{t}}_{+}$and let $\tilde{\mathfrak{s}}$ be the face in $\tilde{\mathfrak{t}}$ which contains $\tilde{\chi}$ in its interior. Recall that $\tilde{\mathfrak{t}}_{v}$ denotes the cone $v \mathfrak{t}_{+} \cap \tilde{\mathfrak{t}}$. Choose $v \in W_{\text {rel }}$ such that $\tilde{\chi} \in \tilde{\mathfrak{t}}_{v}$, in other words $v^{-1} \tilde{\chi} \in \mathfrak{t}_{+}$, and let $\mathfrak{s}$ be the face of $\mathfrak{t}_{+}$which contains $v^{-1} \tilde{\chi}$ in its interior. Then clearly $\tilde{K}_{\tilde{\mathfrak{s}}}=v K_{\mathfrak{s}} v^{-1} \cap \tilde{K}$, so we can define an equivariant embedding

$$
\phi_{\tilde{\mathfrak{s}}, v}: \tilde{X}_{\tilde{\mathfrak{s}}} \rightarrow X_{\mathfrak{s}}
$$

by sending $\tilde{\pi}_{\tilde{\mathfrak{s}}}(1)$ to $\pi_{\mathfrak{s}}(v)$. (Here $\pi_{\mathfrak{s}}$ denotes the quotient map $K \rightarrow X_{\mathfrak{s}}$.) Now select nonzero rational elements $\tilde{\chi}_{1}, \tilde{\chi}_{2}, \ldots, \tilde{\chi}_{n} \in \tilde{\mathfrak{t}}_{\text {such }}$ that each of the subcones $\tilde{\mathfrak{t}}_{v}$ of $\tilde{\mathfrak{t}}_{+}$ is spanned by an appropriate subcollection. If $\tilde{\mathfrak{t}} \cap \mathfrak{c}=0$, this can be accomplished by selecting one nonzero element on every ray (one-dimensional face) occurring in the cubicle subdivision (2.16). For $k=1,2, \ldots, n$, pick $v_{k} \in W_{\text {rel }}$ such that $\tilde{\chi}_{k} \in \tilde{\mathfrak{t}}_{v_{k}}$. Let $\tilde{\mathfrak{s}}_{k}$ and $\mathfrak{s}_{k}$ be the faces of $\tilde{\mathfrak{t}}$ and $\mathfrak{t}$ such that $\tilde{\chi}_{k} \in \tilde{\mathfrak{s}}_{k}^{\circ}$ and $v_{k}^{-1} \tilde{\chi}_{k} \in \mathfrak{s}_{k}^{\circ}$, respectively. For brevity put $W_{\mathfrak{s}_{k}}=W_{k}, W^{\mathfrak{s}_{k}}=W^{k}$, and $\phi_{\tilde{\mathfrak{s}}_{k}, v_{k}}=\phi_{k}$.

3.3.1. Theorem. Let $(\tilde{\lambda}, \lambda) \in \tilde{\mathfrak{t}}_{+}^{*} \times \mathfrak{t}_{+}^{*}$. Then $(\tilde{\lambda}, \lambda) \in \Delta\left(T^{*} K\right)$ if and only if

$$
\left\langle\tilde{w}^{-1} \tilde{\lambda}+f^{*}\left(w^{-1} \lambda\right), \tilde{\chi}_{k}\right\rangle \geq 0
$$

for $k=1,2, \ldots, n$ and for all $(\tilde{w}, w) \in \widetilde{W}^{k} \times W^{k}$ such that $\tilde{\sigma}_{\tilde{u}_{k}}$ is contained in $\phi_{k}^{*}\left(\sigma_{u_{k}}\right)$. Here $\tilde{u}_{k} \in \widetilde{W}^{k}$ and $u_{k} \in W^{k}$ denote the shortest representatives of $\tilde{w}_{0} \tilde{w} \widetilde{W}_{k}$ and $w v_{k} W_{k}$, respectively.

For instance, if $\tilde{\mathfrak{t}}_{+}$is contained in $\mathfrak{t}_{+}$and $\tilde{K}$ is semisimple, then we can choose the $\chi_{k}$ to be the fundamental coweights. Then the cohomological conditions in this theorem involve only the minimal partial flag varieties ("Grassmannians") of $\tilde{K}$ and certain partial flag varieties of $K$, and hence the number of inequalities is considerably smaller than in Theorem 3.1.1. In Section 5 we shall see, however, that even the system of inequalities of Theorem 3.3.1 is often overdetermined.

3.4. Duality. The momentum map on $T^{*} K$ has the property that $\Phi(k,-\xi)=$ $-\Phi(k, \xi)$. This implies immediately that $\Delta\left(T^{*} K\right)$ is stable under the duality involution, which sends $(\tilde{\lambda}, \lambda)$ to $\left(-\tilde{w}_{0} \tilde{\lambda},-w_{0} \lambda\right)$. Consider the inequality (3.2) associated with a triple $(\tilde{w}, w, v) \in \tilde{W} \times W \times W_{\text {rel }}$. Substituting $\tilde{\lambda} \rightarrow-\tilde{w}_{0} \tilde{\lambda}$ and $\lambda \rightarrow-w_{0} \lambda$ and multiplying both sides by $\tilde{w}_{0}$ we obtain

$$
\tilde{w}_{0} \tilde{w}^{-1} \tilde{w}_{0} \tilde{\lambda} \in-\tilde{w}_{0} f^{*}\left(w^{-1} w_{0} \lambda+v \mathcal{C}\right) .
$$

This motivates the following definition. Let $j: \widetilde{W} \hookrightarrow W$ be the lifting homomorphism associated with the chambers $\mathfrak{t}_{+}$and $\tilde{\mathfrak{t}}_{+}$. (Cf. Remark 2.2.3.)

3.4.1. Definition. The dual of $w \in W$ is $w^{*}=j\left(\tilde{w}_{0}\right) w w_{0}$. The $d u a l$ of $\tilde{w} \in \widetilde{W}$ is $\tilde{w}^{*}=\tilde{w}_{0} w \tilde{w}_{0}$. 
Observe that $\left(w^{*}\right)^{*}=w$ and $\left(\tilde{w}^{*}\right)^{*}=\tilde{w}$. However, the duality map on $W$ is in general not a homomorphism, nor does it preserve the length function on $W$ or map the subgroup $\widetilde{W}$ into itself.

3.4.2. Lemma. The set of compatible Weyl group elements $W_{\text {com }} \subseteq W$ is stable under the duality map. Furthermore $\tilde{\mathfrak{t}}_{w^{*}}=-\tilde{w}_{0} \tilde{\mathfrak{t}}_{w}$ and $f^{*}\left(v^{*} \mathcal{C}\right)=-\tilde{w}_{0} f^{*}(v \mathcal{C})$ for

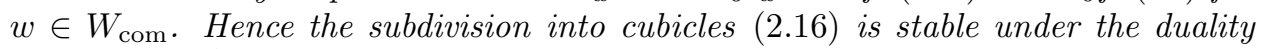
involution of $\tilde{\mathfrak{t}}_{+}$.

Proof. Using the definition of $W_{\text {com }}$ and the fact that $w_{0} \mathfrak{t}_{+}=-\mathfrak{t}_{+}$we find

$$
\begin{aligned}
w \in W_{\text {com }} & \Longleftrightarrow w \mathfrak{t}_{+} \cap \tilde{\mathfrak{t}}_{+}^{\circ} \neq \emptyset \\
& \Longleftrightarrow j\left(\tilde{w}_{0}\right) w w_{0} \mathfrak{t}_{+} \cap \tilde{\mathfrak{t}}_{+}^{\circ} \neq \emptyset \\
& \Longleftrightarrow w^{*} \in W_{\text {com }} .
\end{aligned}
$$

By the same token, $\tilde{\xi} \in \tilde{\mathfrak{t}}_{w^{*}}$ if and only if $\tilde{\xi}=j\left(\tilde{w}_{0}\right) w w_{0} \xi$ for some $\xi \in \mathfrak{t}_{+}$, which is equivalent to $-\tilde{w}_{0} \tilde{\xi}=w\left(-w_{0} \xi\right) \in w \mathfrak{t}_{+} \cap \tilde{\mathfrak{t}}=\tilde{\mathfrak{t}}_{w}$. In other words, $\tilde{\mathfrak{t}}_{w^{*}}=-\tilde{w}_{0} \tilde{\mathfrak{t}}_{w}$. The equality $f^{*}\left(v^{*} \mathcal{C}\right)=-\tilde{w}_{0} f^{*}(v \mathcal{C})$ now follows from Lemma 2.2.4 and the last statement is obvious.

3.4.3. Example. Consider the map $f: \mathbf{S U}(3) \rightarrow \mathbf{U}(15)$ studied in Example 2.3 .2 By (2.22),

$$
j\left(\tilde{w}_{0}\right)=j\left(\tilde{s}_{1} \tilde{s}_{2} \tilde{s}_{1}\right)=(115)(213)(314)(410)(511)(612),
$$

and therefore

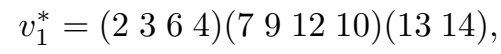

$$
\begin{aligned}
& v_{2}^{*}=1^{*}=(23)(46)(79)(1012)(1314) \text {. }
\end{aligned}
$$

Comparing with 2.23 we see that the duality map does not map $W_{\text {rel }}$ into itself, but for each $v \in W_{\text {rel }}, v^{*} \in \bar{W} v^{\prime}$ for some $v^{\prime} \in W_{\text {rel }}$.

Together with (3.11) Lemma 3.4.2 yields

$$
\left(\tilde{w}^{-1}\right)^{*} \tilde{\lambda} \in f^{*}\left(-\left(w^{-1}\right)^{*} \lambda+v^{*} \mathcal{C}\right)
$$

which is called the dual inequality associated with (3.2). It can be obtained from (3.2) by replacing the triple $(\tilde{w}, w, v)$ with $\left(\tilde{w}^{*},\left(\left(w^{-1}\right)^{*}\right)^{-1}, v^{*}\right)$. We assert that the dual inequality arises from a homological condition analogous to (3.3), namely

$$
\phi^{*}\left(v^{*} \sigma_{\left(\left(w^{-1}\right)^{*}\right)^{-1} v^{*}}\right)\left(\tilde{c}_{\tilde{w}_{0} \tilde{w}^{*}}\right) \neq 0 .
$$

Indeed,

$$
\begin{aligned}
& \phi^{*}\left(v^{*} \sigma_{\left(\left(w^{-1}\right)^{*}\right)^{-1} v^{*}}\right)\left(\tilde{c}_{\tilde{w}_{0} \tilde{w}^{*}}\right)=\phi^{*}\left(j\left(\tilde{w}_{0}\right) v w_{0} \sigma_{w_{0} w v w_{0}}\right)\left(\tilde{c}_{\tilde{w} \tilde{w}_{0}}\right) \\
& =\phi^{*}\left(v w_{0} \sigma_{w_{0} w v w_{0}}\right)\left(\tilde{w}_{0} \tilde{c}_{\tilde{w} \tilde{w}_{0}}\right) \\
& =(-1)^{l(w v)+l\left(\tilde{w}_{0}\right)-l(\tilde{w})} \phi^{*}\left(v \sigma_{w v}\right)\left(\tilde{c}_{\tilde{w}_{0} \tilde{w}}\right),
\end{aligned}
$$

where we have used the $\widetilde{W}$-equivariance of $\phi$ (see Corollary 2.1.5) and Lemma 3.4.4 below. This shows that (3.3) implies (3.13), which explains the appearance of the dual inequality (3.12). 
3.4.4. Lemma. $w_{0} c_{w}=(-1)^{l(w)} c_{w_{0} w w_{0}}$ and $w_{0} \sigma_{w}=(-1)^{l(w)} \sigma_{w_{0} w w_{0}}$.

Proof. The second equality is immediate from the first. By Remark A.1.1, to prove the first equality it is enough to show that $c_{w}^{w_{0}}=(-1)^{l(w)} c_{w}$. We use the notation of that remark. The opposite or dual Borel subgroup is $B^{w_{0}}=w_{0} B w_{0}$. Let $\theta: G \rightarrow G$ be complex conjugation with respect to the real form $K$; then $\theta$ maps $B$ to its opposite and therefore induces an antiholomorphic map $G / B \rightarrow G / B^{w_{0}}$, which we also denote by $\theta$. If $Y$ is a complex submanifold of $G / B$, then so is $\theta(Y)$, but $\theta$ changes the orientation if the complex dimension of $Y$ is odd. It is clear that the diagram

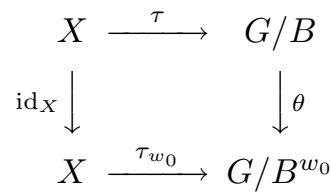

commutes. Moreover, $\theta\left(X_{w}^{\circ}\right)=\left(X_{w}^{w_{0}}\right)^{\circ}$ and therefore

$$
\left(\tau_{w_{0}}\right)_{*}\left(c_{w}\right)=\theta_{*} \tau_{*}\left(c_{w}\right)=\theta_{*}\left(\left[X_{w}\right]\right)=(-1)^{l(w)}\left[X_{w}^{w_{0}}\right]=(-1)^{l(w)}\left(\tau_{w_{0}}\right)_{*} c_{w}^{w_{0}},
$$

which shows that $c_{w}^{w_{0}}=(-1)^{l(w)} c_{w}$.

\section{Semistability}

This section contains the proofs of Theorems 3.1.1 and 3.3.1 In Sections 4.1 and 4.2 we review the Hilbert-Mumford criterion and calculate Mumford's numerical measure of instability for flag varieties. We then finish the proof in Section 4.3. The underlying idea is that an integral coadjoint orbit is a complex projective variety in a natural way, so we can detect points in its momentum polytope by determining the semistable set with respect to various ample line bundles. This is achieved by means of the Hilbert-Mumford criterion, and the upshot is that the semistable set is nonempty if and only if a certain translate of a Schubert cell in the flag variety $X$ intersects the small flag variety $\tilde{X}$. By a transversality argument this leads to inequalities in terms of intersections of Schubert cycles.

Throughout this section the complexifications of the groups $K$ and $\tilde{K}$ are denoted by $G$ and $\tilde{G}$, respectively.

4.1. Measure of instability. The following material is extracted from Mumford's book [20]. Let $Y$ be a complex projective $G$-variety (where $G=K^{\mathbb{C}}$ ) and let $\mathcal{L}$ be an ample $G$-equivariant line bundle over $Y$. Recall that $y \in Y$ is called semistable with respect to $\mathcal{L}$ if for some $n>0$ there exists an invariant section $s$ of $\mathcal{L}^{n}$ such that $s(y) \neq 0$. A point is unstable if it is not semistable. The set of semistable points is denoted by $Y^{\mathrm{ss}}$. It is clearly Zariski-open and $G$-stable.

Let $y$ be any point in $Y$ and let $\chi \in \operatorname{Hom}\left(\mathbb{C}^{\times}, G\right)$ be an algebraic one-parameter subgroup of $G$. Mumford's numerical measure of instability is the integer $m^{\mathcal{L}}(y, \chi)$ determined as follows. Consider $y_{0}=\lim _{t \rightarrow 0} \chi(t) y$, which exists because $Y$ is projective. It is a fixed point for the action of $\chi$ and so $\chi$ acts on the fibre $\mathcal{L}_{y_{0}}$. Let $r$ be the unique integer such that $\chi(t) l=t^{r} l$ for $l \in \mathcal{L}_{y_{0}}$. Then $m^{\mathcal{L}}(y, \chi)=r$. (This sign convention is opposite to Mumford's, but agrees with our sign convention for the moment map.)

4.1.1. Theorem (Hilbert-Mumford criterion). A point $y \in Y$ is semistable if and only if $m^{\mathcal{L}}(y, \chi) \leq 0$ for all one-parameter subgroups $\chi$. 
We will frequently use the following elementary properties of $m^{\mathcal{L}}(y, \chi)$.

4.1.2. Proposition. (i) $m^{\mathcal{L}}\left(g y, g \chi g^{-1}\right)=m^{\mathcal{L}}(y, \chi)$ for all $g \in G$.

(ii) $m^{\mathcal{L}}(p y, \chi)=m^{\mathcal{L}}(y, \chi)$ for all $p \in P_{\chi}$.

(iii) If $\left(Y_{1}, \mathcal{L}_{1}\right)$ and $\left(Y_{2}, \mathcal{L}_{2}\right)$ are two linearized $G$-varieties, then

$$
m^{\mathcal{L}_{1} \otimes \mathcal{L}_{2}}\left(\left(y_{1}, y_{2}\right), \chi\right)=m^{\mathcal{L}_{1}}\left(y_{1}, \chi\right)+m^{\mathcal{L}_{2}}\left(y_{2}, \chi\right)
$$

for $\left(y_{1}, y_{2}\right) \in Y_{1} \times Y_{2}$.

Here $P_{\chi}$ denotes the parabolic subgroup associated with $\chi$, which is defined in (A.1). We identify $\operatorname{Hom}\left(\mathbb{C}^{\times}, H\right)$, the set of algebraic one-parameter subgroups of the complex Cartan $H=T^{\mathbb{C}}$, with the lattice $\Lambda \subseteq \mathfrak{t}$ by assigning to each one-parameter subgroup $\chi$ its infinitesimal generator $d \chi(1)$ times $2 \pi i$. We call $\chi$ dominant if it is contained in $\Lambda_{+}=\Lambda \cap \mathfrak{t}_{+}$and denote by $\langle\xi, \chi\rangle$ the natural pairing between $\chi$ and any $\xi \in \mathfrak{g}^{*}$. According to 4.1.2(i), to calculate $m^{\mathcal{L}}(y, \chi)$ for arbitrary one-parameter subgroups $\chi$ of $G$ it suffices to calculate $m^{\mathcal{L}}(g y, \chi)$ for arbitrary $g \in G$ and dominant one-parameter subgroups $\chi$ of $H$.

4.2. Instability on flag varieties. The following discussion generalizes [20, §4.4]. Take $Y$ to be the flag variety $X=K / T$. We identify $X$ with the complex homogeneous space $G / B$ and consider the homogeneous line bundle

$$
\mathcal{L}_{\lambda}=G \times{ }^{B} \mathbb{C}_{\lambda}
$$

where $\lambda$ is a strictly dominant weight and $\mathbb{C}_{\lambda}$ is the one-dimensional representation of $T$ defined by the weight $\lambda$ (extended to $B$ by letting $H=T^{\mathbb{C}}$ act holomorphically and by letting the unipotent radical of $B$ act trivially). Instead of $m^{\mathcal{L}_{\lambda}}$ we write $m^{\lambda}$. Consider an arbitrary $x \in X$. Let $\pi: G \rightarrow X$ be the quotient map and write $x=\pi(g)$. We assert that if $h \in G$ and $\chi$ is dominant,

$$
m^{\lambda}\left(x, h \chi h^{-1}\right)=m^{\lambda}\left(h^{-1} \pi(g), \chi\right)=\left\langle w^{-1} \lambda, \chi\right\rangle,
$$

where $w$ is the unique Weyl group element for which $\pi(h)$ is in the translated cell $g X_{w}^{\circ}$. Here we use the notation $X_{w}^{\circ}$ for the Bruhat cell $B w B / B \subseteq X$ corresponding to $w \in W$. Indeed, $\pi(h) \in g X_{w}^{\circ}$ is equivalent to $h^{-1} \pi(g)$ being in the $B$-orbit of $\pi\left(w^{-1}\right)$. Since $\chi$ is dominant, $P_{\chi}$ contains $B$, so

$$
m^{\lambda}\left(h^{-1} \pi(g), \chi\right)=m^{\lambda}\left(\pi\left(w^{-1}\right), \chi\right)
$$

by 4.1 .2 (ii). Because $\pi\left(w^{-1}\right)$ is fixed under $H$, we can calculate the right-hand side by considering the action of $\chi$ on the fibre of $\mathcal{L}_{\lambda}=G \times{ }^{B} \mathbb{C}_{\lambda}$ over $\pi\left(w^{-1}\right)$ :

$$
\chi(t)\left[w^{-1}, z\right]=\left[\chi(t) w^{-1}, z\right]=\left[w^{-1},\left(w \chi(t) w^{-1}\right) z\right]=\left[w^{-1}, t^{\left\langle w^{-1} \lambda, \chi\right\rangle} z\right] .
$$

This proves $m^{\lambda}\left(\pi\left(w^{-1}\right), \chi\right)=\left\langle w^{-1} \lambda, \chi\right\rangle$ and hence (4.1).

Evidently, there are no $G$-semistable points on $X$, but the situation becomes interesting when we restrict the action of $G$ to the subgroup $\tilde{G}=\tilde{K}^{\mathbb{C}}$. Again we need only calculate $m^{\lambda}\left(x, \tilde{g} \tilde{\chi} \tilde{g}^{-1}\right)=m^{\lambda}\left(\tilde{g}^{-1} \pi(g), \tilde{\chi}\right)$ for arbitrary $\tilde{g} \in \tilde{G}$ and dominant $\tilde{\chi} \in \operatorname{Hom}\left(\mathbb{C}^{\times}, \tilde{H}\right)$. Now $\tilde{\chi}$ is not necessarily dominant for $G$, but according to (2.16) we can choose $v \in W_{\text {rel }}$ such that $v^{-1} \tilde{\chi}$ is dominant for $G$. Then $m^{\lambda}\left(\tilde{g}^{-1} \pi(g), \tilde{\chi}\right)=$ $m^{\lambda}\left(v^{-1} \tilde{g}^{-1} \pi(g), v^{-1} \tilde{\chi}\right)$, which can be computed by (4.1). The upshot is:

$$
m^{\lambda}\left(x, \tilde{g} \tilde{\chi} \tilde{g}^{-1}\right)=m^{\lambda}\left(\tilde{g}^{-1} \pi(g), \tilde{\chi}\right)=\left\langle f^{*}\left(w^{-1} \lambda\right), \tilde{\chi}\right\rangle
$$

where $w \in W$ is determined by the condition that $\tilde{g} v \in g B w v B$, i.e. $\tilde{g} \pi(v) \in g X_{w v}^{\circ}$. By Proposition 2.2.6(v) the stabilizer of $\pi(v) \in X$ with respect to the $\tilde{G}$-action is 
the Borel $\tilde{B}$. Let $\phi_{v}: \tilde{X} \rightarrow X$ be the $\tilde{G}$-equivariant embedding which sends $\tilde{\pi}(1)$ to $\pi(v)$. Using the Hilbert-Mumford criterion we obtain the following result.

4.2.1. Proposition. Let $\lambda$ be a strictly dominant weight. A point $\pi(g)$ in $X \cong$ $G / B$ is semistable with respect to the bundle $\mathcal{L}_{\lambda}$ and the action of the subgroup $\tilde{G}$ if and only if

$$
\left\langle f^{*}\left(w^{-1} \lambda\right), \tilde{\chi}\right\rangle \leq 0
$$

for all $(w, v) \in W \times W_{\text {rel }}$ and $\tilde{\chi} \in \tilde{\Lambda}$ such that $\tilde{\chi} \in \tilde{\Lambda}_{+} \cap v \Lambda_{+}$and $g X_{w v}^{\circ} \cap \phi_{v}(\tilde{X})$ is nonempty.

If $\lambda$ is dominant but not strictly dominant, then it does not define an ample bundle on $X$, but on the partial flag variety $X_{\mathfrak{s}} \cong G / P$, where $\mathfrak{s}$ is the face of $\mathfrak{t}_{+}$ which contains $\lambda$ in its interior, and $P=P_{\mathfrak{s}}$, the parabolic associated with $\mathfrak{s}$. The analogue of (4.2) is: for all $g$ and dominant $\tilde{\chi}$

$$
m^{\lambda}\left(\tilde{g}^{-1} \pi_{\mathfrak{s}}(g), \tilde{\chi}\right)=\left\langle f^{*}\left(w^{-1} \lambda\right), \tilde{\chi}\right\rangle,
$$

where $w$ is any element of $W$ (unique modulo left multiplication by $W_{\mathfrak{s}}$ ) such that $\tilde{g} v \in g P w v B$, i.e. $\phi_{v}(\tilde{\pi}(\tilde{g})) \in g(P \pi(w v))$. (Note: this condition involves a $P$-orbit in $G / B$, not a $B$-orbit in $G / P$.) By analogy with Proposition 4.2 .1 we obtain the following.

4.2.2. Proposition. Let $\lambda \in \Lambda^{*} \cap \mathfrak{s}^{\circ}$. A point $\pi_{\mathfrak{s}}(g)$ in $X_{\mathfrak{s}} \cong G / P$ is semistable with respect to the bundle $\mathcal{L}_{\lambda}$ and the action of the subgroup $\tilde{G}$ if and only if (4.3) holds for all $(w, v) \in W \times W_{\text {rel }}$ and $\tilde{\chi} \in \tilde{\Lambda}$ such that $\tilde{\chi} \in \tilde{\Lambda}_{+} \cap v \Lambda_{+}$and $g(P \pi(w v)) \cap$ $\phi_{v}(\tilde{X})$ is nonempty.

It is in fact not necessary to verify (4.3) for all dominant one-parameter subgroups of $\tilde{H}$. Choose a collection of dominant one-parameter subgroups $\tilde{\chi}_{1}, \tilde{\chi}_{2}, \ldots$, $\tilde{\chi}_{n}$ of $\tilde{H}$ such that each of the cubicles $\tilde{\mathfrak{t}}_{v}$ in $\tilde{\mathfrak{t}}_{+}$is spanned by an appropriate subcollection. Then every dominant one-parameter subgroup $\tilde{\chi}$ can be written as

$$
\tilde{\chi}=\frac{1}{a} \sum_{k=1}^{n} a_{k} \tilde{\chi}_{k}
$$

where $a$ and $a_{k}$ are integers, $a>0, a_{k} \geq 0$, and $a_{k}=0$ if $\tilde{\chi}_{k}$ is not in the cubicle containing $\tilde{\chi}$. Now let $\lambda \in \Lambda_{+}^{*}$ be strictly dominant. It follows from (4.2) and (4.4) that $\pi(g) \in X$ is semistable for the action of $\tilde{G}$ if and only if $m^{\lambda}\left(\tilde{g}^{-1} \pi(g), \tilde{\chi}_{k}\right) \leq 0$ for all $k$ and for all $\tilde{g} \in \tilde{G}$. For $k=1,2, \ldots, n$, fix $v_{k} \in W_{\text {rel }}$ such that $\tilde{\chi}_{k} \in \tilde{\mathfrak{t}}_{v_{k}}$ (i.e. $v_{k}^{-1} \tilde{\chi}_{k}$ is dominant relative to $G$ ) and let $\tilde{\mathfrak{s}}_{k}$, resp. $\mathfrak{s}_{k}$, be the face in $\tilde{\mathfrak{t}}_{+}$, resp. $\mathfrak{t}_{+}$, which is determined by $\tilde{\chi}_{k} \in \tilde{\mathfrak{s}}_{k}^{\circ}$, resp. $v^{-1} \tilde{\chi}_{k} \in \mathfrak{s}_{k}^{\circ}$. For simplicity let us write $X_{k}=X_{\mathfrak{s}_{k}}, P_{k}=P_{\mathfrak{s}_{k}}, W_{\mathfrak{s}_{k}}=W_{k}, W^{\mathfrak{s}_{k}}=W^{k}$, and $\tilde{\pi}_{\tilde{\mathfrak{s}}_{k}}=\tilde{\pi}_{k}$. Let $\phi_{\tilde{\mathfrak{s}}_{k}, v_{k}}=\phi_{k}$ be the embedding of $\tilde{X}_{k}$ into $X_{k}$ defined in (3.10). Again using 4.1.2(1ii) we get a stronger version of (4.2):

$$
m^{\lambda}\left(\tilde{g}^{-1} \pi(g), \tilde{\chi}_{k}\right)=\left\langle f^{*}\left(w^{-1} \lambda\right), \tilde{\chi}_{k}\right\rangle
$$

where $w \in W^{k}$ is determined by $\tilde{g} v_{k} \in g B w v_{k} P_{k}$, in other words $\phi_{k}\left(\tilde{\pi}_{k}(\tilde{g})\right) \in$ $g\left(X_{k}\right)_{w v_{k}}^{\circ}$. The conclusion is as follows. 
4.2.3. Proposition. Let $\lambda$ be a strictly dominant weight. A point $\pi(g)$ in $X$ is semistable with respect to the bundle $\mathcal{L}_{\lambda}$ and the action of the subgroup $\tilde{G}$ if and only if

$$
\left\langle f^{*}\left(w^{-1} \lambda\right), \tilde{\chi}_{k}\right\rangle \leq 0
$$

for $k=1,2, \ldots, n$ and for all $w \in W^{k}$ such that $g\left(X_{k}\right)_{u_{k}}^{\circ} \cap \phi_{k}\left(\tilde{X}_{k}\right)$ is nonempty. Here $u_{k} \in W^{k}$ denotes the shortest representative of $w v_{k} W_{k}$.

We leave it to the reader to state a version for singular dominant weights.

4.3. Proof of Theorems 3.1.1 and 3.3.1, The proof of Theorem 3.1.1 is in two steps: first we show that Theorem 3.2.4 implies Theorem 3.2.2, and then we establish Theorem 3.2.2. As we pointed out earlier, Theorem 3.2 .2 is equivalent to Theorem 3.1.1, so this will finish the proof.

Step 1. Suppose we knew Theorem 3.2.4 was true. Let us apply it to the pair $(\tilde{K}, \tilde{K} \times K)$ and the inclusion map $\operatorname{id}_{\tilde{K}} \times f$. Consider $(\tilde{\lambda}, \lambda) \in \tilde{\Lambda}_{+}^{*} \times \Lambda_{+}^{*}$ and note that $\left(\tilde{V}_{\tilde{\lambda}} \otimes V_{\lambda}\right)^{\tilde{K}} \neq\{0\}$ if and only if $\tilde{V}_{\tilde{\lambda}}$ is a summand of $V_{\lambda}^{*} \cong V_{-w_{0} \lambda}$. Hence, by Theorem 3.2.3, there exists $n>0$ such that $\tilde{V}_{n \tilde{\lambda}}$ occurs in $V_{-n w_{0} \lambda}$ if and only if

$$
0 \in\left(\operatorname{id}_{\tilde{K}} \times f\right)^{*}\left(\left(\tilde{w}^{-1} \tilde{\lambda}, w^{-1} \lambda\right)-\tilde{\mathcal{C}} \times v \mathcal{C}\right)
$$

for all $(\tilde{w}, w, v)$ such that

$$
\left(\operatorname{id}_{\tilde{X}} \times \phi\right)^{!}\left(\tilde{c}_{\tilde{w}_{0} \tilde{w}}, v c_{w_{0} w v}\right) \neq 0 .
$$

Now $\left(\operatorname{id}_{\tilde{K}} \times f\right)^{*}(\tilde{\mathcal{C}} \times v \mathcal{C})=\tilde{\mathcal{C}}+f^{*}(v \mathcal{C})=f^{*}(v \mathcal{C})$ by Proposition 2.2.6(iii), so (4.5) is equivalent to

$$
\tilde{w}^{-1} \tilde{\lambda} \in f^{*}\left(-w^{-1} \lambda+v \mathcal{C}\right) .
$$

Furthermore, (4.6) is equivalent to

$$
\tilde{c}_{\tilde{w}_{0} \tilde{w}} \cdot \phi^{!}\left(v c_{w_{0} w v}\right) \neq 0
$$

in $H_{2 m}(\tilde{X}, \mathbb{Z})$, where $m=l\left(\tilde{w}_{0}\right)-l(\tilde{w})-l(w v)$. The conclusion is that $\tilde{V}_{n \tilde{\lambda}} \subseteq V_{-n w_{0} \lambda}$ for some $n>0$ if and only if (4.7) holds for all $\tilde{w}, w$ and $v$ satisfying (4.8).

In (4.8) it is actually sufficient to take into account only those triples $(\tilde{w}, w, v)$ for which the intersection is zero-dimensional. To see why, recall that the class $\tilde{c}_{\tilde{w}_{0}} \tilde{w}$ is represented by the Schubert variety $\tilde{X}_{\tilde{w}_{0} \tilde{w}} \subseteq \tilde{X}$ and that $v c_{w_{0} w v}$ is represented by an algebraic cycle $X_{w, v}$ which is a linear combination of Schubert varieties in $X$. Then (4.8) means that for generic $\tilde{g} \in \tilde{K}$ and $g \in K$ the translated cycles $\tilde{g} \tilde{X}_{\tilde{w}_{0}} \tilde{w}$ and $g X_{w, v}$ intersect in an $m$-dimensional cycle on $\tilde{X}$. If $m>0$, this implies they intersect in a boundary component $\tilde{X}_{\tilde{w}_{0} \tilde{u}}^{\circ}$ of $\tilde{X}_{\tilde{w}_{0} \tilde{w}}$ because of the fact that $\tilde{X}_{\tilde{w}_{0} \tilde{w}}^{\circ}$ is an affine variety. In other words, we have $\tilde{c}_{\tilde{w}_{0} \tilde{u}} \cdot \phi^{!}\left(v c_{w_{0} w v}\right) \neq 0$ for some $\tilde{u} \succcurlyeq \tilde{w}$ (where $\succcurlyeq$ denotes the Bruhat-Chevalley order), and hence

$$
\tilde{u}^{-1} \tilde{\lambda} \in f^{*}\left(-w^{-1} \lambda+v \mathcal{C}\right) .
$$

Because $\tilde{\lambda}$ is dominant, $\tilde{u}^{-1} \tilde{\lambda} \leq \tilde{w}^{-1} \tilde{\lambda}$, so if (4.9) holds, then

$$
\tilde{w}^{-1} \tilde{\lambda} \in \tilde{u}^{-1} \tilde{\lambda}+\tilde{\mathcal{C}} \subseteq f^{*}\left(-w^{-1} \lambda+v \mathcal{C}\right)+\tilde{\mathcal{C}}=f^{*}\left(-w^{-1} \lambda+v \mathcal{C}\right) .
$$

This shows that the inequality (4.9) is stronger than (4.7). By induction we can discard all inequalities (4.8) except those for which $m=0$, in which case they are equivalent to $\phi^{*}\left(v \sigma_{w v}\right)\left(\tilde{c}_{\tilde{w}_{0}} \tilde{w}\right) \neq 0$. Thus we see (after replacing $\lambda$ by $-w_{0} \lambda$ and $w$ by $\left.w_{0} w\right)$ that Theorem 3.2 .4 implies Theorem 3.2 .2 
Step 2. We now prove Theorem 3.2.4. Consider $\lambda \in \Lambda_{+}^{*}$. Let us assume first that $\lambda$ is strictly dominant. Let $\mathcal{L}_{\lambda}$ be the homogeneous ample line bundle on $X \cong G / B$ introduced in Section 4.2 The Borel-Weil Theorem says that the space of holomorphic sections of $\mathcal{L}_{\lambda}^{n}$ is isomorphic to $V_{n \lambda}$ as a $K$-module. Hence, by definition, there exists $n>0$ such that $V_{n \lambda}$ contains an invariant vector if and only if $X^{\mathrm{ss}}$ is nonempty. By Proposition 4.2.1 this is equivalent to the existence of a $g \in G$ such that

$$
0 \in f^{*}\left(w^{-1} \lambda+v \mathcal{C}\right)
$$

for all $(w, v) \in W \times W_{\text {rel satisfying }}$

$$
g X_{w v}^{\circ} \cap \phi_{v}(\tilde{X}) \neq \emptyset .
$$

Here we have used that $f^{*}(v \mathcal{C})$ is dual to the cone spanned by $\tilde{\Lambda}_{+} \cap v \Lambda_{+}$, which follows from Lemma 2.2.4. Since the semistable set is Zariski-open, by Kleiman's transversality theorem (see e.g. Theorem 10.8 in [11, Ch. III]) we can perturb $g$ to move $g X_{w v}^{\circ}$ into general position with respect to $\phi_{v}(\tilde{X})$. Then (4.11) amounts to $\phi_{v}^{!}\left(c_{w v}\right) \neq 0$. Now observe that the holomorphic embedding $\phi_{v}: \tilde{X} \rightarrow X$ is equal to the composition $v^{-1} \circ \phi$ (even though $v^{-1}$ is not holomorphic). Hence

$$
0 \neq \phi_{v}^{!}\left(c_{w v}\right)=\left(v^{-1} \circ \phi\right)^{!}\left(c_{w v}\right)=\phi^{!}\left(v^{-1}\right)^{!}\left(c_{w v}\right)=(-1)^{l(v)} \phi^{!}\left(v c_{w v}\right) .
$$

Substituting $\lambda \rightarrow-w_{0} \lambda$ in (4.10), $w \rightarrow w_{0} w$ in (4.10) and (4.12), and using that $V_{n \lambda}$ contains a $\tilde{K}$-invariant vector if and only if $V_{n \lambda}^{*} \cong V_{-n w_{0} \lambda}$ does, we obtain Theorem 3.2 .4 for strictly dominant integral $\lambda$.

The case where $\lambda \in \Lambda_{+}^{*}$ is dominant but not strictly dominant is very similar. We briefly point out the main differences. Instead of $X$ we use the partial flag variety $X_{\mathfrak{s}}=K / K_{\mathfrak{s}} \cong G / P$ with the $G$-equivariant line bundle $\mathcal{L}_{\lambda}=G \times{ }^{P} \mathbb{C}_{\lambda}$. Here $\mathfrak{s}$ is the face of $\mathfrak{t}_{+}$such that $\lambda \in \mathfrak{s}^{\circ}, P=P_{\mathfrak{s}}$ is the parabolic subgroup of $G$ attached to $\mathfrak{s}$, and $\mathbb{C}_{\lambda}$ is the obvious $P$-module defined by $\lambda$. For any $w \in W$ the closure of the $P$-orbit through $\pi(w)$ is a single Schubert variety, namely $X_{w^{\prime}}$, where $w^{\prime}$ is the longest element in the coset $W_{\mathfrak{s}} w \subseteq W$. The same reasoning as above (using Proposition 4.2.2 instead of 4.2.1) now gives that $\lambda \in \Delta\left(T^{*}(\tilde{K} \backslash K)\right.$ ) if and only if 4.10 holds for all $w$ and $v$ satisfying

$$
\phi^{!}\left(v c_{w^{\prime} v}\right) \neq 0 .
$$

But if (4.10) holds for $w$, it holds for all elements in the coset $W_{\mathfrak{s}} w$, because $W_{\mathfrak{s}}$ fixes $\lambda$. This finishes the proof of Theorem 3.2.4.

The proof of Theorem 3.3.1 is almost exactly the same, but uses Proposition 4.2 .3 instead of 4.2 .1 The details are left to the reader.

\section{EXAMPLES}

5.1. Kostant's theorem. As a simple illustration of Theorem 3.2 .1 we consider $\tilde{K}=T$, the maximal torus of $K$. The answer here is of course well-known and can be obtained in many other ways. Observe that $\tilde{T}=T, \tilde{W}=\{1\}$, and every element of $\mathfrak{t}$ is "dominant" with respect to $T$. Therefore $W_{\text {rel }}=W, \tilde{\mathfrak{t}}_{v}=v \mathfrak{t}_{+}$, and the decomposition (2.16) is simply the partition of $\mathfrak{t}$ into Weyl chambers, $\mathfrak{t}=\bigcup_{v \in W} v \mathfrak{t}_{+}$. The dual cone to $v \mathfrak{t}_{+}$is $v \mathcal{C}$, where $\mathcal{C}$ is the root cone of $K$. The flag variety $\tilde{X}$ is a point, so $\phi^{*}$ is the trivial homomorphism $H \bullet(X, \mathbb{Z}) \rightarrow \mathbb{Z}$. Since $\phi^{*}$ preserves degree, we see that $\phi^{*}\left(v \sigma_{w v}\right) \neq 0$ if and only if $v=w^{-1}$. In other words, for every $\lambda \in \mathfrak{t}_{+}^{*}$ the points $\tilde{\lambda}$ in the polytope $\Delta\left(\mathcal{O}_{\lambda}\right)$ are described by the inequalities $w \tilde{\lambda} \in \lambda-\mathcal{C}$, 
where $w$ ranges over $W$. This is equivalent to Kostant's result that $\Delta\left(\mathcal{O}_{\lambda}\right)$ is the convex hull of the Weyl group orbit $W \lambda$.

5.2. Klyachko's theorem. In this section we apply our main theorem to the diagonal embedding of a group into a number of copies of itself. We deviate from our standard notation and denote the small group by $K$ and the large group $K \times$ $K \times \cdots \times K$ ( $m$ times) by $K^{m}$. The canonical projection $f^{*}:\left(\mathfrak{t}^{*}\right)^{m} \rightarrow \mathfrak{t}^{*}$ is the addition map. There is a canonical embedding of the small Weyl group $W$ into the large Weyl group $W^{m}$, namely the diagonal embedding. This tallies with Example 2.1.6 because every regular point in the diagonal subgroup $K \hookrightarrow K^{m}$ is regular relative to $K^{m}$. For the same reason, $W_{\text {rel }}=\{1\}$ and the partition (2.16) has only one piece, namely $\mathfrak{t}_{1}=\mathfrak{t}_{+}$. Its dual cone is $\mathcal{C}$, the root cone of $K$. Moreover, the large flag variety is $X^{m}$ and the embedding $\phi: X \rightarrow X^{m}$ is the diagonal map. The induced homomorphism $\phi^{*}: H^{\bullet}(X, \mathbb{Z})^{\otimes m} \cong H^{\bullet}\left(X^{m}, \mathbb{Z}\right) \rightarrow H^{\bullet}(X, \mathbb{Z})$ is the cup product, and the Gysin map

$$
\phi^{!}: H_{i}\left(X^{m}, \mathbb{Z}\right) \rightarrow H_{i-(m-1) l\left(w_{0}\right)}(X, \mathbb{Z})
$$

is the intersection product. Similarly, for every face $\mathfrak{s}$ of $\mathfrak{t}_{+}$the embedding (3.10) is simply the diagonal embedding of the partial flag variety $X_{\mathfrak{s}}$ into the $m$-fold product $X_{\mathfrak{s}}^{m}$. Now choose a collection of vectors $\chi_{1}, \chi_{2}, \ldots, \chi_{n} \in \mathfrak{t}$ which span the cone $\mathfrak{t}_{+}$. Then Theorem 3.3.1 comes down to the following.

5.2.1. Theorem. Let $\left(\lambda_{1}, \lambda_{2}, \ldots, \lambda_{m+1}\right) \in\left(\mathfrak{t}_{+}^{*}\right)^{m+1}$. Then $\left(\lambda_{1}, \lambda_{2}, \ldots, \lambda_{m+1}\right) \in$ $\Delta\left(\left(T^{*} K\right)^{m}\right)$ if and only if

$$
\sum_{l=1}^{m+1}\left\langle w_{l}^{-1} \lambda_{l}, \chi_{k}\right\rangle \geq 0
$$

for $k=1,2, \ldots, n$ and for all $\left(w_{1}, w_{2}, \ldots, w_{m+1}\right) \in W^{k} \times W^{k} \times \cdots \times W^{k}$ such that $\sigma_{u_{k}}$ is contained in $\sigma_{w_{1}} \cup \sigma_{w_{2}} \cup \cdots \cup \sigma_{w_{m}}$. Here $u_{k} \in W^{k}$ denotes the shortest representative of $w_{0} w_{m+1} W_{k}$.

For $K=\mathbf{U}(n)$ this theorem was proved by Klyachko [15]. Also for $K=\mathbf{U}(n)$, Helmke and Rosenthal [13] proved that the inequalities (5.1) are necessary, but not that they are sufficient. Inequalities for eigenvalues of sums of Hermitian matrices have a long history going back to Weyl; see the cited papers for examples. The essential case of the theorem is of course when $K$ is semisimple; the identity component of the centre just contributes a number of equalities, e.g. for the trace when $K=\mathbf{U}(n)$. The cup product condition is closely related to the LittlewoodRichardson rules, which are discussed in Fulton [8, 9], Pragacz [21] and Littelmann [18.

5.2.2. Example $\left(\mathbf{G}_{2} \times \mathbf{G}_{2}\right)$. Let us work out the case $K=\mathbf{G}_{2}$ and $m=2$. Denote by $S=\left\{\alpha_{1}, \alpha_{2}\right\}$ the simple roots of $\mathbf{G}_{2}$, where $\alpha_{1}$ is short and $\alpha_{2}$ is long. Let $s_{1}=s_{\alpha_{1}}$ and $s_{2}=s_{\alpha_{2}}$ be the corresponding simple reflections and $\pi_{1}=2 \alpha_{1}+\alpha_{2}$ and $\pi_{2}=3 \alpha_{1}+2 \alpha_{2}$ the fundamental weights. For $k=1$ or 2 , let $P_{k}$ be the parabolic attached to $\pi_{k}, W_{k}$ the Weyl group, $W^{k}$ the set of shortest representatives for $W / W_{k}$, and $X_{k}$ the associated ten-dimensional "Grassmannian". Then

$$
\begin{array}{ll}
W_{1}=\left\{1, s_{2}\right\}, & W^{1}=\left\{1, s_{1}, s_{2} s_{1}, s_{1} s_{2} s_{1},\left(s_{2} s_{1}\right)^{2}, s_{1}\left(s_{2} s_{1}\right)^{2}\right\}, \\
W_{2}=\left\{1, s_{1}\right\}, & W^{2}=\left\{1, s_{2}, s_{1} s_{2}, s_{2} s_{1} s_{2},\left(s_{1} s_{2}\right)^{2}, s_{2}\left(s_{1} s_{2}\right)^{2}\right\} .
\end{array}
$$


Using Chevalley's formula, Theorem A.2.1(iii), we can compute multiplication tables for the cohomology of $X_{k}$ in terms of the Schubert bases. We find the relations

$$
\begin{aligned}
& \sigma_{s_{1}}^{2}=\sigma_{s_{2} s_{1}}, \quad \sigma_{s_{1}}^{3}=2 \sigma_{s_{1} s_{2} s_{1}}, \quad \sigma_{s_{1}}^{4}=2 \sigma_{\left(s_{2} s_{1}\right)^{2}}, \quad \sigma_{s_{1}}^{5}=2 \sigma_{s_{1}\left(s_{2} s_{1}\right)^{2}} \quad \text { for } k=1, \\
& \sigma_{s_{2}}^{2}=3 \sigma_{s_{1} s_{2}}, \quad \sigma_{s_{2}}^{3}=6 \sigma_{s_{2} s_{1} s_{2}}, \quad \sigma_{s_{2}}^{4}=18 \sigma_{\left(s_{1} s_{2}\right)^{2}}, \quad \sigma_{s_{2}}^{5}=18 \sigma_{s_{2}\left(s_{1} s_{2}\right)^{2}} \quad \text { for } k=2 \text {, }
\end{aligned}
$$

from which it is easy to derive all triples $\left(w_{1}, w_{2}, w_{3}\right) \in W^{k} \times W^{k} \times W^{k}$ such that $\sigma_{u_{k}}$ is contained in $\sigma_{w_{1}} \cup \sigma_{w_{2}}$. For $k=1$ they are:

$$
\begin{gathered}
\left(1,1, s_{1}\left(s_{2} s_{1}\right)^{2}\right), \quad\left(1, s_{1},\left(s_{2} s_{1}\right)^{2}\right), \quad\left(1, s_{2} s_{1}, s_{1} s_{2} s_{1}\right), \\
\left(s_{1}, s_{1}, s_{1} s_{2} s_{1}\right), \quad\left(s_{1}, s_{2} s_{1}, s_{2} s_{1}\right)
\end{gathered}
$$

plus permutations of these triples. For $k=2$ we obtain the same list, but with $s_{1}$ and $s_{2}$ interchanged. If we identify $\mathfrak{g}_{2}^{*}$ with $\mathfrak{g}_{2}$ by means of the invariant inner product for which $\left\|\alpha_{1}\right\|^{2}=2$, then we can choose the $\chi_{k}$ to be the fundamental weights $\pi_{k}$. The inequalities for the polygons $\Delta\left(\mathcal{O}_{\lambda_{1}} \times \mathcal{O}_{\lambda_{2}}\right)$ are best written out in coordinates relative to the basis $\left\{\pi_{1}, \pi_{2}\right\}$, because then the positive chamber is given by the positive quadrant. Writing $\lambda_{i}=x_{i} \pi_{1}+y_{i} \pi_{2}$, we get for $k=1$

$$
\begin{aligned}
2 x_{1}+3 y_{1}+2 x_{2}+3 y_{2}-2 x_{3}-3 y_{3} & \geq 0 \\
2 x_{1}+3 y_{1}+x_{2}+3 y_{2}-x_{3}-3 y_{3} & \geq 0 \\
2 x_{1}+3 y_{1}+x_{2}-x_{3} & \geq 0 \\
x_{1}+3 y_{1}+x_{2}+3 y_{2}-x_{3} & \geq 0 \\
x_{1}+3 y_{1}+x_{2}+x_{3} & \geq 0
\end{aligned}
$$

and for $k=2$

$$
\begin{array}{r}
x_{1}+2 y_{1}+x_{2}+2 y_{2}-x_{3}-2 y_{3} \geq 0 \\
x_{1}+2 y_{1}+x_{2}+y_{2}-x_{3}-y_{3} \geq 0 \\
x_{1}+2 y_{1}+y_{2}-y_{3} \geq 0 \\
x_{1}+y_{1}+x_{2}+y_{2}-y_{3} \geq 0 \\
x_{1}+y_{1}+y_{2}+y_{3} \geq 0
\end{array}
$$

up to permutations of the variables. The triples $\left(s_{1}, s_{2} s_{1}, s_{2} s_{1}\right)$ and $\left(s_{2}, s_{1} s_{2}, s_{1} s_{2}\right)$ (the associated cycles of which have intersection multiplicity $>1$ ) turn out to be redundant. The inequalities associated with the triples containing a 1 express the fact that

$$
\Delta\left(\mathcal{O}_{\lambda_{1}} \times \mathcal{O}_{\lambda_{2}}\right) \subseteq\left(\lambda_{1}+\text { hull } W \lambda_{2}\right) \cap\left(\lambda_{2}+\text { hull } W \lambda_{1}\right) .
$$

Figure 3 shows an example where this inclusion is strict. The dark shading indicates the moment polygon and the light shading its Weyl group translates. The dotted lines are the polygons $\lambda_{1}+$ hull $W \lambda_{2}$ and $\lambda_{2}+$ hull $W \lambda_{1}$.

5.3. $\mathfrak{s l}(2)$-triples. Our next example is a general homomorphism with finite kernel $f: \tilde{K} \rightarrow K$, where $\tilde{K}=\mathbf{S U}(2)$ and $K$ is semisimple. Up to conjugacy such homomorphisms are in one-to-one correspondence with embeddings of $\tilde{\mathfrak{g}}=\mathfrak{s l}(2, \mathbb{C})$ into $\mathfrak{g}$, that is to say, triples $(\tilde{h}, \tilde{e}, \tilde{f})$ of vectors in $\mathfrak{g}$ which satisfy

$$
[\tilde{h}, \tilde{e}]=2 \tilde{e}, \quad[\tilde{h}, \tilde{f}]=-2 \tilde{f}, \quad[\tilde{e}, \tilde{f}]=\tilde{h} .
$$

Indeed, because $\mathbf{S L}(2, \mathbb{C})$ is simply connected any such embedding lifts to a homomorphism $\mathbf{S L}(2, \mathbb{C}) \rightarrow G$, which can be conjugated to a homomorphism that maps 


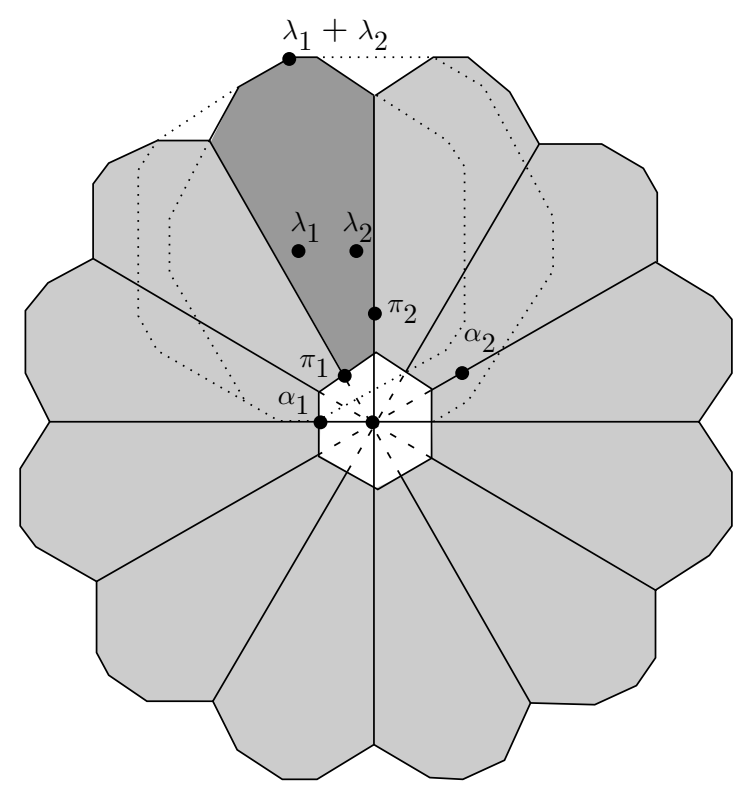

Figure 3. Moment "rosette" of $\mathcal{O}_{\lambda_{1}} \times \mathcal{O}_{\lambda_{2}}$, where $K=\mathbf{G}_{2}, \lambda_{1}=$ $\frac{1}{2}\left(5 \pi_{1}+\pi_{2}\right)$, and $\lambda_{2}=\frac{1}{2}\left(\pi_{1}+3 \pi_{2}\right)$

$\mathbf{S U}(2)$ into $K$, since $K$ is maximal compact in $G$. In other words, we may assume that $\tilde{h} \in \mathfrak{t}, \tilde{e} \in \mathfrak{n}$ and $\tilde{f} \in \theta \mathfrak{n}$. After a further conjugation with an element of $W$ we may even assume that $\tilde{h} \in \mathfrak{t}_{+}$. Then the chambers $\tilde{\mathfrak{t}}_{+}$and $\mathfrak{t}_{+}$are compatible and $W_{\text {rel }}=\{1\}$.

Let $\left\{\alpha^{*} \mid \alpha \in S\right\}$ be the set of fundamental coweights, i.e. the basis of $\mathfrak{t}$ which is dual to $S \subseteq \mathfrak{t}^{*}$. Then we can write

$$
\tilde{h}=\sum_{\alpha \in S \backslash \bar{S}} d_{\alpha} \alpha^{*}
$$

where all $d_{\alpha}>0$ and $\bar{S} \subseteq S$ is the set of simple roots orthogonal to $\tilde{h}$. According to Dynkin's classification of $\mathfrak{s l}(2)$-triples (see [7]), $d_{\alpha}=1$ or 2 for all $\alpha \notin \bar{S}$. The set $\bar{S}$ is a base of the root system $\bar{R}$ of $\bar{K}=\mathcal{Z}_{K}(\tilde{T})$, and $\bar{W}$ is generated by the simple reflections $s_{\alpha}$ with $\alpha \in \bar{S}$. Let $j: \widetilde{W}=\left\{1, \tilde{w}_{0}\right\} \rightarrow W$ be the inclusion map determined by the choice of dominant chambers $\tilde{\mathfrak{t}}_{+}$and $\mathfrak{t}_{+}$. This map is easy to describe explicitly.

5.3.1. Lemma. (i) $w_{0} \tilde{h}=-\tilde{h}$.

(ii) $j\left(\tilde{w}_{0}\right)=w_{0} \bar{w}_{0}$.

(iii) $w_{0} \bar{w}_{0}$ is an involution.

Proof. The $\widetilde{W}$-equivariance of the embedding $f_{*}: \tilde{\mathfrak{t}} \rightarrow \mathfrak{t}$ implies that $j\left(\tilde{w}_{0}\right) \tilde{h}=-\tilde{h}$, which is in the antidominant chamber $-\mathfrak{t}_{+}$. On the other hand, $w_{0} \tilde{h}$ is also in $-\mathfrak{t}_{+}$ and hence $w_{0} \tilde{h}=-\tilde{h}$. This proves (ii).

To prove (iii) it suffices to show that $w_{0} \bar{w}_{0}$ is in $\mathcal{N}_{W}(\bar{S})$ and maps $\tilde{h}$ to $-\tilde{h}$. It follows from (ii) that $w_{0}$ preserves $\bar{R}$, and therefore sends $\bar{R}_{+}$to $\bar{R}_{-}$. Consequently $w_{0} \bar{w}_{0}$ preserves $\bar{S}$. Since $\bar{W}$ fixes $\tilde{h}, w_{0} \bar{w}_{0} \tilde{h}=w_{0} \tilde{h}=-\tilde{h}$ by (i).

(iii) follows immediately from (iii). 
In order to write the inequalities for the "polytopes" $\Delta\left(\mathcal{O}_{\lambda}\right)$ we identify $\tilde{\mathfrak{t}}^{*}$ with $\mathbb{R}$ by sending the positive root $\tilde{\alpha}$ to 2 . Dually, this corresponds to sending the basis element $\tilde{h} \in \tilde{\mathfrak{t}}$ to 1 . The projection $f^{*}(\lambda) \in \tilde{\mathfrak{t}}^{*}$ of any functional $\lambda \in \mathfrak{t}^{*}$ then gets identified with the number $\lambda(\tilde{h})$.

5.3.2. Proposition. Let $\lambda \in \mathfrak{t}_{+}^{*}$. Then $\tilde{\lambda} \in \Delta\left(\mathcal{O}_{\lambda}\right)$ if and only if $\tilde{\lambda} \geq 0$ and

$$
-\lambda(\tilde{h})+\max _{\alpha \in S \backslash \bar{S}} d_{\alpha} \lambda\left(\alpha^{\vee}\right) \leq \tilde{\lambda} \leq \lambda(\tilde{h}) .
$$

Proof. Following Theorem 3.2 .1 we determine all $(\tilde{w}, w) \in \widetilde{W} \times W$ such that $\phi^{*}\left(\sigma_{w}\right)\left(\tilde{c}_{\tilde{w}}\right) \neq 0$, i.e. $\tilde{\sigma}_{\tilde{w}}$ is contained in $\phi^{*}\left(\sigma_{w}\right)$. Since $\tilde{X}=\mathbb{C} P^{1}, H^{2 l(\tilde{w})}(\tilde{X}, \mathbb{Z})$ vanishes for $l(\tilde{w})>1$. Moreover, $\phi^{*}$ preserves degree, so that we need only consider Weyl group elements of length $\leq 1$.

For $\tilde{w}=w=1$ we find $\tilde{\sigma}_{\tilde{w}}=\sigma_{w}=1$ and get the inequality $\tilde{\lambda} \leq \lambda(\tilde{h})$.

If $l(\tilde{w})=l(w)=1$, then $\tilde{w}=\tilde{w}_{0}$ and $w=s_{\alpha}$ for some $\alpha \in S$. Using Theorem A.2.1 we find that $\phi^{*}\left(\sigma_{s_{\alpha}}\right)=f^{*}\left(\pi_{\alpha}\right) \tilde{\sigma}_{\tilde{w}_{0}}=\pi_{\alpha}(\tilde{h}) \tilde{\sigma}_{\tilde{w}_{0}}$, where $\pi_{\alpha}$ denotes the fundamental weight corresponding to $\alpha$. Therefore $\tilde{\sigma}_{\tilde{w}_{0}}$ is contained in $\phi^{*}\left(\sigma_{s_{\alpha}}\right)$ if and only if $\pi_{\alpha}(\tilde{h}) \neq 0$. From (5.2) we obtain

$$
\pi_{\alpha}(\tilde{h})=\sum_{\beta \in S \backslash \bar{S}} d_{\beta} \pi_{\alpha}\left(\beta^{*}\right) .
$$

Since the angle between any two fundamental weights $\pi_{\alpha}$ and $\pi_{\beta}$ is acute, we have $\pi_{\alpha}\left(\beta^{*}\right) \geq 0$ for all $\alpha$ and $\beta$, and therefore $\pi_{\alpha}(\tilde{h}) \neq 0$ if and only if $\pi_{\alpha}\left(\beta^{*}\right) \neq 0$ for some $\beta \in S \backslash \bar{S}$. This is certainly the case if $\alpha \in S \backslash \bar{S}$, because then we can take $\beta=\alpha$, but may also happen if $\alpha \in \bar{S}$. In any case, the inequality corresponding to $\left(\tilde{w}_{0}, s_{\alpha}\right)$ is $\tilde{w}_{0} \tilde{\lambda} \leq f^{*}\left(s_{\alpha} \tilde{h}\right)=\lambda\left(s_{\alpha} \lambda\right)$, or

$$
\tilde{\lambda} \geq-\lambda\left(s_{\alpha} \tilde{h}\right)=-\lambda(\tilde{h})+\lambda\left(\alpha^{\vee}\right) \alpha(\tilde{h}) .
$$

Here we have used that $\tilde{w}_{0} \tilde{\lambda}=-\tilde{\lambda}$ and $s_{\alpha} \lambda=\lambda-\lambda\left(\alpha^{\vee}\right) \alpha$. Moreover,

$$
\alpha(\tilde{h})=\sum_{\beta \in S \backslash \bar{S}} d_{\beta} \alpha\left(\beta^{*}\right)= \begin{cases}d_{\alpha} & \text { if } \alpha \notin \bar{S} \\ 0 & \text { if } \alpha \in \bar{S}\end{cases}
$$

Thus, for $\alpha \in \bar{S}$ we obtain the inequality $\tilde{\lambda} \geq-\lambda(\tilde{h})$, which is vacuous, and for $\alpha \notin \bar{S}$ we obtain $\tilde{\lambda} \geq-\lambda(\tilde{h})+\lambda\left(\alpha^{\vee}\right) d_{\alpha}$.

5.3.3. Example. There exists a triple $(\tilde{h}, \tilde{e}, \tilde{f})$, known as the principal triple, for which $\bar{S}=\emptyset$ and $d_{\alpha}=2$ for all $\alpha$. It was shown by Dynkin that principal triples are unique up to conjugation. In this case we get the inequalities $-\lambda(\tilde{h})+2 \max _{\alpha \in S} \lambda\left(\alpha^{\vee}\right) \leq \tilde{\lambda} \leq \lambda(\tilde{h})$. For instance, if $K$ is the product of $m$ copies of $\mathbf{S U}(2)$, then the principal $\mathbf{S U}(2)$ is the diagonal subgroup. Here $\lambda$ can be represented as an $m$-tuple $\left(\tilde{\lambda}_{1}, \tilde{\lambda}_{2}, \ldots, \tilde{\lambda}_{m}\right)$ and the inequalities are

$$
-\tilde{\lambda}_{1}-\tilde{\lambda}_{2}-\cdots-\tilde{\lambda}_{m}+2 \max _{i} \tilde{\lambda}_{i} \leq \tilde{\lambda} \leq \tilde{\lambda}_{1}+\tilde{\lambda}_{2}+\cdots+\tilde{\lambda}_{m} .
$$

This can of course also be regarded as a special case of Theorem [5.2.1. 
5.4. A maximal rank subgroup. Let $K=\mathbf{G}_{2}$. The long roots form a subsystem of the root system of $K$ and the associated subgroup $\tilde{K}$ is isomorphic to $\mathbf{S U}(3)$. Using the notation of Example 5.2.2, we denote the simple roots of $K$ by $\alpha_{1}$ and $\alpha_{2}$ and its fundamental weights by $\pi_{1}$ and $\pi_{2}$. Writing $\tilde{\alpha}_{1}$ and $\tilde{\alpha}_{2}$ for the simple roots of $\tilde{K}$, and $\tilde{\pi}_{1}$ and $\tilde{\pi}_{2}$ for its fundamental weights, we have $\alpha_{1}=\frac{1}{3}\left(\tilde{\alpha}_{1}-\tilde{\alpha}_{2}\right)$, $\alpha_{2}=\tilde{\alpha}_{2}, \pi_{1}=\tilde{\pi}_{1}$ and $\pi_{2}=\tilde{\pi}_{1}+\tilde{\pi}_{2}$. The matrix of $f^{*}$ relative to the fundamental weights is therefore

$$
f^{*}=\left(\begin{array}{ll}
1 & 1 \\
0 & 1
\end{array}\right)
$$

The dominant chamber of $K$ consists of two chambers of $\tilde{K}$, and the relative Weyl set is $\left\{1, s_{1}\right\}$. According to Theorem 3.3.1 we need to consider three oneparameter subgroups $\tilde{\chi}_{1}, \tilde{\chi}_{2}, \tilde{\chi}_{3}$, and corresponding embeddings $\phi_{1}: \tilde{G} / \tilde{P}_{1} \rightarrow G / P_{1}$, $\phi_{2}: \tilde{G} / \tilde{B} \rightarrow G / P_{2}$ and $\phi_{3}: \tilde{G} / \tilde{P}_{2} \rightarrow G / P_{1}$. However, the inequalities coming from $\phi_{3}$ are dual to those of $\phi_{1}$, so we need to consider only $\phi_{1}$ and $\phi_{2}$. The cohomology of the Grassmannians of $K$ was computed in Example 5.2.2. The calculation for $\tilde{K}$ is very similar. By means of (5.3) and Theorem A.2.1 we can then calculate all pairs $(\tilde{w}, w) \in \widetilde{W}^{k} \times W^{k}$ such that $\tilde{\sigma}_{\tilde{w}}$ is contained in $\phi_{k}^{*}\left(\sigma_{w}\right)$. For $k=1$ we find

$$
(1,1), \quad\left(\tilde{s}_{1}, s_{1}\right), \quad\left(\tilde{s}_{2} \tilde{s}_{1}, s_{2} s_{1}\right),
$$

and for $k=2$,

$$
(1,1), \quad\left(\tilde{s}_{1}, s_{2}\right), \quad\left(\tilde{s}_{2}, s_{2}\right), \quad\left(\tilde{s}_{1} \tilde{s}_{2}, s_{1} s_{2}\right), \quad\left(\tilde{s}_{2} \tilde{s}_{1}, s_{1} s_{2}\right), \quad\left(\tilde{w}_{0}, s_{2} s_{1} s_{2}\right) .
$$

In fact, for all these pairs $\tilde{\sigma}_{\tilde{w}}$ occurs with multiplicity 1 in $\phi_{k}^{*}\left(\sigma_{w}\right)$. Introducing coordinates $\tilde{\lambda}=\tilde{x} \tilde{\pi}_{1}+\tilde{y} \tilde{\pi}_{2}$ and $\lambda=x \pi_{1}+y \pi_{2}$, we can then write the inequalities $\left\langle\tilde{w}^{-1} \tilde{\lambda}, \tilde{\chi}_{k}\right\rangle \leq\left\langle w^{-1} \lambda, \chi_{k}\right\rangle$ as follows. It turns out that the inequalities for $k=1$ are all redundant, as are their dual inequalities (which are obtained by interchanging $\tilde{x}$ and $\tilde{y})$. For $k=2$ the pairs $\left(\tilde{s}_{1} \tilde{s}_{2}, s_{1} s_{2}\right)$ and $\left(\tilde{s}_{2} \tilde{s}_{1}, s_{1} s_{2}\right)$ lead to redundant inequalities. The remaining ones are

$$
\tilde{x}+\tilde{y} \leq x+2 y, \quad \tilde{x} \leq x+y, \quad \tilde{y} \leq x+y, \quad \tilde{x}+\tilde{y} \geq y .
$$

This set of inequalities is self-dual (stable under interchanging $\tilde{x}$ and $\tilde{y}$ ). In addition to the obvious inequalities $\tilde{x} \geq 0, \tilde{y} \geq 0, x \geq 0, y \geq 0$, these completely describe the set of all $(\tilde{\lambda}, \lambda) \in \tilde{\mathfrak{t}}_{+}^{*} \times \mathfrak{t}_{+}^{*}$ such that $\tilde{\lambda} \in \Delta\left(\mathcal{O}_{\lambda}\right)$. See Figure 4 for examples.
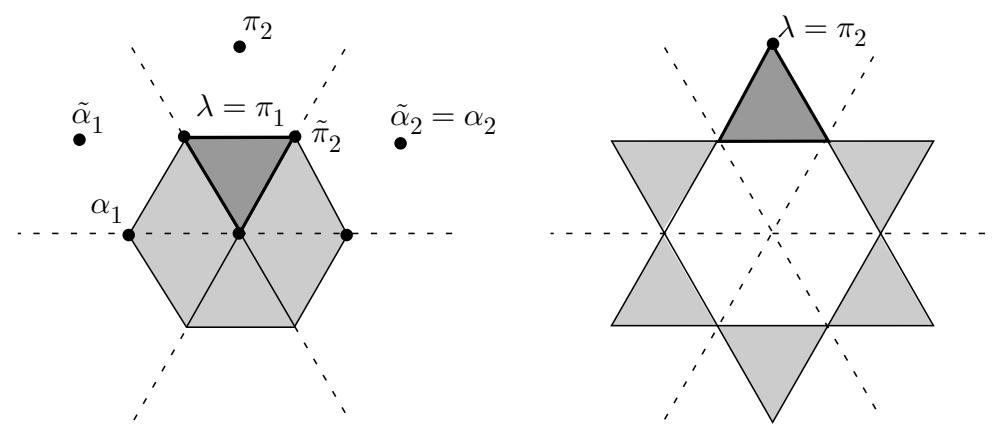

Figure 4. Moment rosettes of $\mathbf{G}_{2}$-orbits $\mathcal{O}_{\lambda}$ w.r.t. $\mathbf{S U}(3)$-action, where $\lambda=\pi_{1}$, resp. $\lambda=\pi_{2}$ (complexified adjoint representation) 


\section{Appendix A. Flag VARIETIES}

A.1. Schubert cells. Let $G=K^{\mathbb{C}}$ be the complexification of $K$ and $H=T^{\mathbb{C}}$ the complexified maximal torus. Let $R \subseteq \mathfrak{t}^{*}$ be the root system of $K$ and $R_{+}$the set of positive roots. Let $\mathfrak{n}$ be the nilpotent subalgebra of $\mathfrak{g}$ spanned by the positive root spaces and $N=\exp \mathfrak{n}$ the corresponding maximal unipotent subgroup of $G$. Furthermore, let $\mathfrak{b}$ be the Borel subalgebra $\mathfrak{h} \oplus \mathfrak{n}$ and $B=\exp \mathfrak{b}$ the corresponding Borel subgroup. The canonical map

$$
\tau: X=K / T \rightarrow G / B
$$

defined by $\tau(k T / T)=k B / B$ is a $K$-equivariant diffeomorphism. The complex homogeneous space $G / B$ decomposes into Bruhat cells $X_{w}^{\circ}=B w B / B$, where $w \in W$. The Schubert variety $X_{w}$ is the closure of $X_{w}^{\circ}$; the Schubert class is the fundamental class $\left[X_{w}\right] \in H_{2 l(w)}(G / B, \mathbb{Z})$, where $l(w)$ is the length of $w$. Define

$$
c_{w}=\tau_{*}^{-1}\left(\left[X_{w}\right]\right) ;
$$

these classes form the Schubert basis of the homology group $H_{\bullet}(X, \mathbb{Z})$.

A.1.1. Remark. The Schubert basis of $H \cdot(X, \mathbb{Z})$ depends on the choice of the set of positive roots. Let us work out the formula for a change of basis. Any set of positive roots can be written as $u R_{+}$for a unique $u \in W$, and the Borel associated with $u R_{+}$is $B^{u}=u B u^{-1}$. Let us write $\left(X_{w}^{u}\right)^{\circ}$ for the Schubert cell $B^{u} w B^{u} / B^{u}$ in $G / B^{u}, \tau_{u}$ for the canonical $K$-equivariant diffeomorphism $X \rightarrow G / B^{u}$, and $c_{w}^{u}$ for the homology class $\left(\tau_{u}\right)_{*}^{-1}\left(\left[X_{w}^{u}\right]\right)$. Consider the commutative diagram

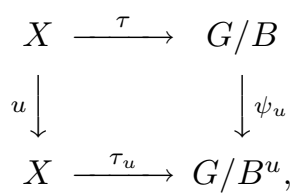

where the vertical arrow on the left denotes the action of $u$ on $X$ and the map $\psi_{u}$ is the $G$-equivariant holomorphic map $\psi_{u}(g B / B)=g u^{-1} B^{u} / B^{u}$. It is clear that $\psi_{u}\left(X_{w}^{\circ}\right)=u^{-1}\left(X_{u w u^{-1}}^{u}\right)^{\circ}$, so we see that

$$
\left(\tau_{u}\right)_{*}\left(u c_{w}\right)=\left(\psi_{u}\right)_{*} \tau_{*}\left(c_{w}\right)=\left(\psi_{u}\right)_{*}\left(\left[X_{w}\right]\right)=\left[X_{u w u^{-1}}^{u}\right]=\left(\tau_{u}\right)_{*} c_{u w u^{-1}}^{u} .
$$

We conclude that the Schubert basis relative to the set of positive roots $u R_{+}$is given by $c_{w}^{u}=u c_{u^{-1} w u}$.

If $P$ is any parabolic subgroup of $G$, we have the partial flag variety $G / P$ and a $K$-equivariant diffeomorphism $\tau: K /(P \cap K) \rightarrow G / P$. We call $P$ standard if $P \supseteq B$; then $P=G_{\mathfrak{s}} N$, where $G_{\mathfrak{s}}$ is the complexification of $K_{\mathfrak{s}}=\mathcal{Z}_{K}(\mathfrak{s})$, the centralizer of a face $\mathfrak{s} \subseteq \mathfrak{t}_{+}$. In this case we write $P=P_{\mathfrak{s}}$ and $K / K_{\mathfrak{s}}=X_{\mathfrak{s}}$. Every parabolic is of the form

$$
P_{\chi}=\left\{p \in G \mid \lim _{t \rightarrow 0} \chi(t) p \chi(t)^{-1} \text { exists }\right\}
$$

for some algebraic one-parameter subgroup $\chi \in \operatorname{Hom}\left(\mathbb{C}^{\times}, G\right)$, and we have $P_{\chi}=P_{\mathfrak{s}}$ if and only if (the infinitesimal generator of) $\chi$ is in $\mathfrak{s}^{\circ}$. The Schubert cells in $G / P$ 
are the sets $\left(X_{\mathfrak{s}}\right)_{w}^{\circ}=B w P / P$; their closures are the Schubert varieties $\left(X_{\mathfrak{s}}\right)_{w}$, and the classes $c_{w}^{\mathfrak{s}}=\tau_{*}^{-1}\left(\left[\left(X_{\mathfrak{s}}\right)_{w}\right]\right)$ form the Schubert basis of $H_{\bullet}\left(X_{\mathfrak{s}}, \mathbb{Z}\right)$. Here $w$ ranges over the subset $W^{\mathfrak{s}}$ of $W$.

A.2. Cohomology. Consider the $\operatorname{map} H^{\bullet}(X, \mathbb{Z}) \rightarrow H^{\bullet}(\tilde{X}, \mathbb{Z})$ induced by the embedding $\phi: \tilde{X} \rightarrow X$. Note that $X=[K, K] /(T \cap[K, K])$ and that $f$ maps $[\tilde{K}, \tilde{K}]$ into $[K, K]$. Hence $\phi$ can also be viewed as the embedding induced by the homomorphism $[\tilde{K}, \tilde{K}] \rightarrow[K, K]$. For the purpose of this discussion we may therefore assume both $K$ and $\tilde{K}$ to be semisimple.

Define a map $\Theta: \Lambda^{*} \rightarrow H^{2}(X, \mathbb{Z})$ by $\Theta(\lambda)=c_{1}\left(\mathcal{L}_{\lambda}\right)$, the first Chern class of the homogeneous line bundle with weight $\lambda$. See [3] or [6] for the theorems quoted below.

A.2.1. Theorem (Chevalley). (i) $\Theta$ is an isomorphism.

(ii) $\Theta\left(\pi_{\alpha}\right)=\sigma_{s_{\alpha}}$ for all simple roots $\alpha$.

(iii) For all weights $\lambda$

$$
\Theta(\lambda) \cup \sigma_{w}=\sum_{\substack{\beta \in R_{+} \\ l\left(w s_{\beta}\right)=l(w)+1}} \lambda\left(\beta^{\vee}\right) \sigma_{w s_{\beta}} .
$$

Parts (ii) and (ii) lead to the following simple description of the map $\phi^{*}$ restricted to $H^{2}$. It is clear that the diagram

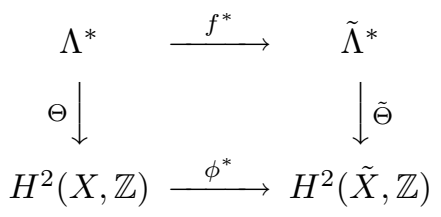

commutes. In degree 2 , the matrix of $\phi^{*}$ relative to the Schubert bases is therefore the same as the matrix of $f^{*}$ relative to the bases of fundamental weights.

Now consider the graded algebra $S\left(\mathfrak{t}^{*}\right)$ of polynomial functions on $\mathfrak{t}$ (in which elements of $\mathfrak{t}^{*}$ are defined to be of degree 2) and the ideal $J$ generated by the $W$-invariant polynomials of positive degree. Recall the following well-known result.

A.2.2. Theorem (Borel). The map $\Theta$ extends to a surjective homomorphism of graded algebras $S\left(\mathfrak{t}^{*}\right) \rightarrow H^{*}(X, \mathbb{R})$, whose kernel is equal to $J$.

Observe that $f^{*}(J) \subseteq \tilde{J}$, so that the map $\phi^{*}$ can be alternatively described (at least over $\mathbb{R}$ ) as the homomorphism induced by the restriction map $S\left(\mathfrak{t}^{*}\right) \rightarrow$ $S\left(\tilde{\mathfrak{t}}^{*}\right)$. Thus the degree-2 Schubert classes $\sigma_{s_{\alpha}}$ generate the cohomology of $X$ over $\mathbb{R}$ (though not over $\mathbb{Z}$ ), and by using Theorem A.2.1 (iii) one can in principle calculate the matrix of $\phi^{*}$ in higher degrees. This can be laborious in practice, however.

A convenient method for calculating the Weyl group action on the cohomology is provided by the difference operators $D_{\alpha}: S\left(\mathfrak{t}^{*}\right) \rightarrow S\left(\mathfrak{t}^{*}\right)$, which are defined for $\alpha \in R$ by

$$
D_{\alpha}(p)=\frac{p-s_{\alpha}(p)}{\alpha}
$$

where $p \in S\left(\mathfrak{t}^{*}\right)$. It is clear that $D_{\alpha}(J)=\{0\}$. Hence, by Theorem A.2.2, $D_{\alpha}$ induces a linear operator of degree -2 on $H^{*}(X, \mathbb{R})$, which will also be denoted by $D_{\alpha}$. Note that $D_{\alpha}^{2}=0$ for all $\alpha$. 
A.2.3. Theorem (Bernstein et al. [3, Demazure [6]). For all simple roots $\alpha$ and all $w \in W$ we have

$$
D_{\alpha}\left(\sigma_{w}\right)= \begin{cases}0 & \text { if } w s_{\alpha} \succ w, \\ \sigma_{w s_{\alpha}} & \text { if } w s_{\alpha} \prec w .\end{cases}
$$

Thus $s_{\alpha} \sigma_{w}=\sigma_{w}-\Theta(\alpha) D_{\alpha}\left(\sigma_{w}\right)$, which is equal to $\sigma_{w}$ if $w s_{\alpha} \succ w$ and to $\sigma_{w}-\Theta(\alpha) \cup \sigma_{w s_{\alpha}}$ if $w s_{\alpha} \prec w$.

\section{Appendix B. Notation}

$K ; T ; C$

$G ; H$

$W ; w_{0}$

$R ; R_{+}$

$S ; \mathcal{C}$

$N ; B$

$\mathcal{N}_{I}(J) ; \mathcal{Z}_{I}(J)$

$X ; \pi$

$\mathfrak{t}_{+} ; \mathfrak{t}_{+}^{*}$

$\Lambda ; \Lambda^{*}$

$\Lambda_{+} ; \Lambda_{+}^{*}$

$\mathcal{O}_{\lambda}$

$f ; f^{*}$

$X_{w}^{\circ} ; X_{w}$

$c_{w} ; \sigma_{w}$

$\mathcal{P}$

$\phi ; \phi^{!}$

$\mathfrak{s} ; \mathfrak{s}^{\circ}$

$K_{\mathfrak{s}} ; R_{\mathfrak{s}}$

$W_{\mathfrak{s}} ; W^{\mathfrak{s}}$

$P_{\mathfrak{s}} ; X_{\mathfrak{s}}$

$\pi_{\mathfrak{s}} ; \phi_{\tilde{\mathfrak{s}}}$

$\mathcal{L}_{\lambda}$

$P_{\chi}$

$\left(X_{\mathfrak{s}}\right)_{w}^{\circ} ;\left(X_{\mathfrak{s}}\right)_{w}$

$c_{w}^{\mathfrak{s}}$

$\tilde{\mathfrak{t}}_{w} ; W_{\text {rel }}$ compact connected Lie group; maximal torus; centre

complexification of $K$; resp. $T$

Weyl group of $K$ w.r.t. $T$; longest Weyl group element

root system; positive roots

simple roots; cone spanned by $S$

maximal unipotent subgroup of $G$; Borel subgroup $H N$

normalizer; resp. centralizer of group $J$ in a group $I$

full flag variety $K / T \cong G / B$; projection $G \rightarrow X$

positive Weyl chamber in $\mathfrak{t}$; positive Weyl chamber in $\mathfrak{t}^{*}$

integral lattice in $\mathfrak{t}$; weight lattice $\operatorname{Hom}_{\mathbb{Z}}(\Lambda, \mathbb{Z})$

dominant one-parameter subgroup; dominant characters

coadjoint orbit through $\lambda \in \mathfrak{k}^{*}$

homomorphism $\tilde{K} \rightarrow K$; dual projection $\mathfrak{k}^{*} \rightarrow \tilde{\mathfrak{k}}^{*}$

Bruhat cell $B w B / B \subseteq X$; its closure

Schubert cycle $\left[X_{w}\right]$ in $H_{2 l(w)}(X, \mathbb{Z})$; cocycle in $H^{2 l(w)}(X, \mathbb{Z})$

Poincaré duality $H^{i}(X, \mathbb{Z}) \rightarrow H_{2 l\left(w_{0}\right)-i}(X, \mathbb{Z})$

embedding $\tilde{X} \rightarrow X$; wrong-way map $\tilde{\mathcal{P}} \circ \phi^{*} \circ \mathcal{P}^{-1}$

face of $\mathfrak{t}_{+} ;$its relative interior

centralizer of $\mathfrak{s}$; its root system

Weyl group of $K_{\mathfrak{s}}$; set of shortest representatives of $W / W_{\mathfrak{s}}$ parabolic $G_{\mathfrak{s}} N$; partial flag variety $K / K_{\mathfrak{s}} \cong G / P_{\mathfrak{s}}$

projection $G \rightarrow X_{\mathfrak{s}}$; inclusion $\tilde{X}_{\tilde{\mathfrak{s}}} \rightarrow X_{\mathfrak{s}}$ (if $\tilde{K}_{\tilde{\mathfrak{s}}} \subseteq K_{\mathfrak{s}}$ )

line bundle $G \times{ }^{P_{\mathfrak{s}}} \mathbb{C}_{\lambda}$ on $X_{\mathfrak{s}}$ (for $\lambda \in \mathfrak{s}^{\circ} \cap \Lambda^{*}$ )

parabolic associated with one-parameter subgroup $\chi$ of $G$

Bruhat cell $B w P / P \subseteq X_{\mathfrak{s}}$; its closure

Schubert cycle $\left[\left(X_{\mathfrak{s}}\right)_{w}\right]$ in $H_{2 l(w)}\left(X_{\mathfrak{s}}, \mathbb{Z}\right)\left(\right.$ for $\left.w \in W^{\mathfrak{s}}\right)$

cone $w \mathfrak{t}_{+} \cap \tilde{\mathfrak{t}}($ for $w \in W$ ); relative Weyl set

Similar conventions are in force for the groups $\tilde{K}$ and $\bar{K}$, i.e. $\tilde{C}$ and $\bar{C}$ denote the centres of $\tilde{K}$ and $\bar{K}, \tilde{G}$ and $\bar{G}$ their complexifications, etc. We assume furthermore that $f(\tilde{T}) \subseteq T$ and $f(\tilde{B}) \subseteq B$. Here $\tilde{K}$ is an arbitrary compact connected Lie group, $f: \tilde{K} \rightarrow K$ is a homomorphism with finite kernel, and $\bar{K}=\mathcal{Z}_{K}(f(\tilde{T}))$.

Weights, roots. We identify the character group $\operatorname{Hom}\left(T, S^{1}\right)$ with the weight lattice $\Lambda^{*}$ by mapping a character $\lambda$ to the weight $d \lambda(1) / 2 \pi i$. We identify $\mathfrak{c}^{*}$ with the annihilator of $[\mathfrak{k}, \mathfrak{k}]$ in $\mathfrak{k}^{*}$ and $[\mathfrak{k}, \mathfrak{k}]^{*}$ with the annihilator of $\mathfrak{c}$, so that we have a direct sum decomposition $\mathfrak{k}^{*}=\mathfrak{c}^{*} \oplus[\mathfrak{k}, \mathfrak{k}]^{*}$.

The roots are the weights of the complexified adjoint representation $\mathfrak{g}$. Over $\mathbb{R}$ they span the subspace $\mathfrak{t}^{*} \cap[\mathfrak{k}, \mathfrak{k}]^{*}$ of $\mathfrak{t}^{*}$. Given a base $S$ of $R$, the set of fundamental coweights is the basis $\left\{\alpha^{*} \mid \alpha \in S\right\}$ of $\mathfrak{t} \cap[\mathfrak{k}, \mathfrak{k}]$ which is dual to $S$. The dual root 
system $R^{\vee}$ is the root system in $\mathfrak{t} \cap[\mathfrak{k}, \mathfrak{k}]$ consisting of the dual roots or coroots $\alpha^{\vee}$, which are determined by $s_{\alpha} \lambda=\lambda-\lambda\left(\alpha^{\vee}\right) \alpha$. The set of fundamental weights is the basis $\left\{\pi_{\alpha} \mid \alpha \in S\right\}$ of $\mathfrak{t}^{*} \cap[\mathfrak{k}, \mathfrak{k}]^{*}$ which is dual to $S^{\vee}$.

If we identify $\mathfrak{t}$ with $\mathfrak{t}^{*}$ via a $W$-invariant inner product $\langle\cdot, \cdot\rangle$, then $\alpha^{\vee}=2 \alpha /\langle\alpha, \alpha\rangle$ and $\alpha^{*}=2 \pi_{\alpha} /\langle\alpha, \alpha\rangle$.

The Weyl chamber $\mathfrak{t}_{+}$is equal to $\mathfrak{c} \times \operatorname{cone}\left\{\alpha^{*} \mid \alpha \in S\right\}$, whereas $\mathfrak{t}_{+}^{*}$ is equal to $\mathfrak{c}^{*} \times \operatorname{cone}\left\{\pi_{\alpha} \mid \alpha \in S\right\}$. (Here $\operatorname{cone}(X)$ denotes the set of all nonnegative linear combinations of elements in $X$.) We define the root cone to be the cone $\mathcal{C}$ spanned by $R_{+}$; it is dual to the cone $\mathfrak{t}_{+}$in the sense that $\lambda \in \mathcal{C}$ if and only if $\lambda(\xi) \geq 0$ for all $\xi \in \mathfrak{t}_{+}$.

\section{REFERENCES}

[1] S. Agnihotri and C. Woodward, Eigenvalues of products of unitary matrices and quantum Schubert calculus, Math. Res. Lett. 5 (1998), no. 6, 817-836. MR 2000a:14066

[2] P. Belkale, Local systems on $\mathbb{P}^{1}-S$ for $S$ a finite set, preprint, University of Chicago, 1999.

[3] I. Bernstein, I. Gelfand, and S. Gelfand, Schubert cells and the cohomology of the spaces $G / P$, Uspehi Mat. Nauk 28 (1973), no. 3(171), 3-26 (Russian), English translation in Russian Math. Surveys 28 (1973), no. 3, 1-26. MR 55:2941

[4] N. Bourbaki, Groupes et algèbres de Lie, Éléments de mathématique, Masson, Paris, 1982. MR 84i:22001

[5] M. Brion, On the general faces of the moment polytope, Internat. Math. Res. Notices (1999), no. 4, 185-201. CMP 99:09

[6] M. Demazure, Désingularisation des variétés de Schubert généralisées, Ann. Sci. École Norm. Sup. (4) 7 (1974), 53-88. MR 50:7174

[7] E. B. Dynkin, Semisimple subalgebras of semisimple Lie algebras, Mat. Sbornik N. S. 30(72) (1952), 349-462 (Russian), English translation in American Mathematical Society Translations, Series 2, vol. 6, Amer. Math. Soc., Providence, R.I., 1957, pp. 111-244. MR 13:904c

[8] W. Fulton, Young tableaux, London Mathematical Society Student Texts, vol. 35, Cambridge University Press, Cambridge, 1997. MR 99f:05119

[9] _ Eigenvalues of sums of Hermitian matrices (after A. Klyachko), Séminaire Bourbaki, 50ème année, exposé no. 845 (Paris, 1997-98), Astérisque, vol. 252, Société Mathématique de France, Paris, 1998, pp. 255-269. CMP 99:13

[10] V. Guillemin and S. Sternberg, Convexity properties of the moment mapping, Invent. Math. 67 (1982), no. 3, 491-513. MR 83m:58037

[11] R. Hartshorne, Algebraic geometry, Graduate Texts in Mathematics, vol. 52, Springer-Verlag, New York, 1977. MR 57:3116

[12] G. J. Heckman, Projections of orbits and asymptotic behavior of multiplicities for compact connected Lie groups, Invent. Math. 67 (1982), no. 2, 333-356. MR 84d:22019

[13] U. Helmke and J. Rosenthal, Eigenvalue inequalities and Schubert calculus, Math. Nachr. 171 (1995), 207-225. MR 96b:15039

[14] F. Kirwan, Convexity properties of the moment mapping, III, Invent. Math. 77 (1984), no. 3, 547-552. MR 86b:58042b

[15] A. Klyachko, Stable bundles, representation theory and Hermitian operators, Selecta Math. (N.S.) 4 (1998), no. 3, 419-445. MR 2000b:14054

[16] A. Knutson and T. Tao, The honeycomb model of $\mathrm{GL}_{n}(\mathbf{C})$ tensor products. I. Proof of the saturation conjecture, J. Amer. Math. Soc. 12 (1999), no. 4, 1055-1090. CMP 99:15

[17] M. Krämer, Über Untergruppen kompakter Liegruppen als Isotropiegruppen bei linearen Aktionen, Math. Z. 147 (1976), no. 3, 207-224. MR 53:8330]

[18] P. Littelmann, A generalization of the Littlewood-Richardson rule, J. Algebra 130 (1990), no. 2, 328-368. MR 91f:22023

[19] P.-L. Montagard, Sur les faces du cône associé au pléthysme, Comm. Algebra 26 (1998), no. 7, 2321-2336. MR 99e:20057

[20] D. Mumford, J. Fogarty, and F. Kirwan, Geometric invariant theory, third ed., Ergebnisse der Mathematik und ihrer Grenzgebiete, 2. Folge, vol. 34, Springer-Verlag, Berlin, 1994. MR 95m:14012 
[21] P. Pragacz, Algebro-geometric applications of Schur $S$ - and Q-polynomials, Topics in Invariant Theory (Paris, 1989-1990) (M.-P. Malliavin, ed.), Lecture Notes in Mathematics, vol. 1478, Springer-Verlag, Berlin, 1991, pp. 130-191. MR 93h:05170

[22] R. Sjamaar, Convexity properties of the moment mapping re-examined, Adv. Math. 138 (1998), no. 1, 46-91. MR 2000a:53148

Department of Mathematics, Cornell University, Ithaca, New York 14853-4201

Current address: Department of Mathematics, Harvard University, Cambridge, Massachusetts 02138-2901

E-mail address: arkadiy@math.harvard.edu

Department of Mathematics, Cornell University, Ithaca, New York 14853-4201

E-mail address: sjamaar@math.cornell.edu 